\title{
RAPID SIMULATION OF SOLID DEPOSITION IN CRYOGENIC HEAT EXCHANGERS TO IMPROVE RISK MANAGEMENT IN LNG PRODUCTION
}

\author{
Corey J. Baker, Jordan H. Oakley, Darren Rowland, Thomas J. Hughes ${ }^{\dagger}$, Zachary M. \\ Aman, and Eric F. May* \\ Fluid Science \& Resources Division, ARC Training Centre for LNG Futures, \\ The University of Western Australia \\ Crawley, WA, AUSTRALIA
}

\section{Highlights}

- A new tool is presented for assessing deposition risk in LNG production

- Matching literature data, the tool represents an operational freeze-out incident well

- Risk assessment reveals possible mitigation strategies and future research needs

\begin{abstract}
The carryover of heavy hydrocarbons into cryogenic heat exchangers, even at low concentrations, represents a significant risk to reliable LNG production given their propensity to freeze, deposit and block flow. Calculating blockage risk requires not only freeze-out temperature predictions but also estimates of where solids will form and the associated operational symptoms. We present here a new simulation tool capable of such predictions in industrial cryogenic heat exchangers, which has been tuned to solid formation data available in the literature. Simulation of the LNG plant upset detailed by Ismail and Al Thani (LNG-18, 2016) confirms the location of the initial deposition that occurred upon re-start, and provides insights into possible remediation strategies that could have helped avoid the subsequent shut down. The analysis also reveals that predicting water crystallisation in cryogenic LNG systems requires a substantial extrapolation of current thermodynamic models, highlighting the need for definitive measurements under such conditions.

*Corresponding author: eric.may@uwa.edu.au

${ }^{\dagger}$ Current address: Oil \& Gas Engineering, Monash University, Clayton VIC, AUSTRALIA
\end{abstract}

Keywords: LNG production; risk assessment; cryogenic heat exchangers; solid solubility; solid deposition; numerical simulation. 


\section{Graphical Abstract}
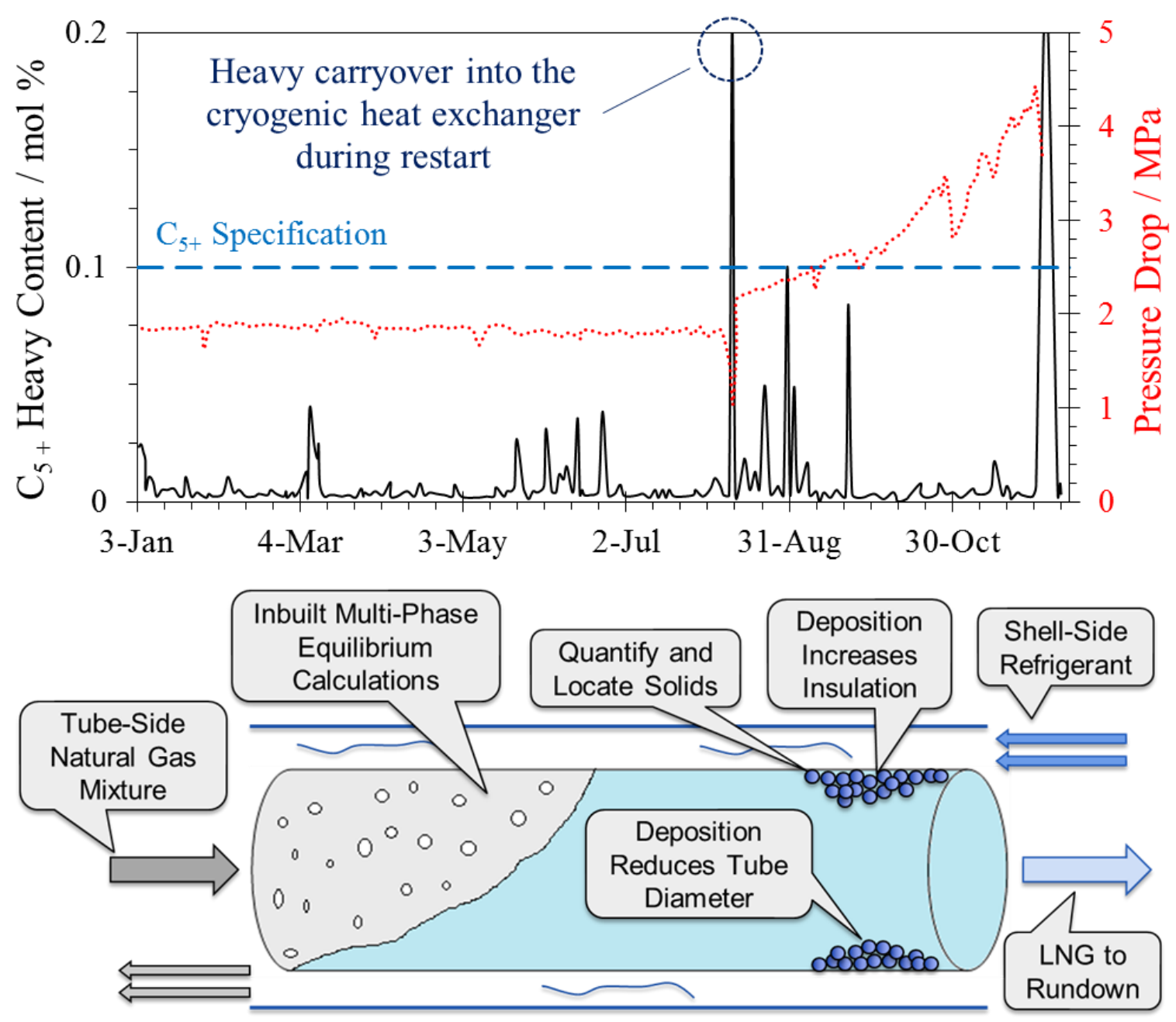


\section{Introduction}

Natural gas accounts for nearly $24 \%$ of world energy production, with approximately 32 $\%$ of global trade occurring through the production and transport of Liquefied Natural Gas (LNG) [1]. So-called 'mega-scale' production trains with capacities around 1 to 8 million tonnes per annum generate the vast majority of the LNG traded internationally, with volumes on the order of $200,000 \mathrm{~m}^{3}$ being shipped on a weekly basis from such plants [2-4]. The regularity of this shipment schedule is often central to the business model of mega-scale LNG production: decade-long supply contracts enable customers to satisfy their needs for energy security and diversity, while helping producers to secure the investment capital needed for plant construction and operation. Accordingly, the financial penalties associated with missing just one scheduled LNG shipment are significant given that, at the current spot price of US\$6 per GJ [5], the value of the LNG cargo is around US\$30 million. The severity of this consequence means that the risk associated with an unplanned shut-down of a mega-scale LNG plant is appreciable, and it is essential that the probability of technical problems capable of causing such an outcome be minimised.

Before natural gas is liquefied, impurities such as acid gases, water and heavy hydrocarbons must be removed [6]. A cryogenic distillation column (also known as an LNG scrub column) is used to separate hydrocarbons based on their volatility. A majority of the heavier components $\left(\mathrm{C}_{3+}\right.$ fraction) exit through the bottom outlet for processing in a liquefied petroleum gas (LPG) fractionation train, and the lighter hydrocarbons (rich in methane) are sent overhead to the main cryogenic heat exchanger (MCHE) for conversion to LNG [7, 8], as shown in Figure 1. The gas is cooled in the MCHE to about $122 \mathrm{~K}\left(-151^{\circ} \mathrm{C}\right)$ producing a high pressure LNG, which then enters a turbine or a flash expansion valve to provide further cooling to about $112 \mathrm{~K}\left(-161^{\circ} \mathrm{C}\right)$ at (or near) atmospheric pressure. 


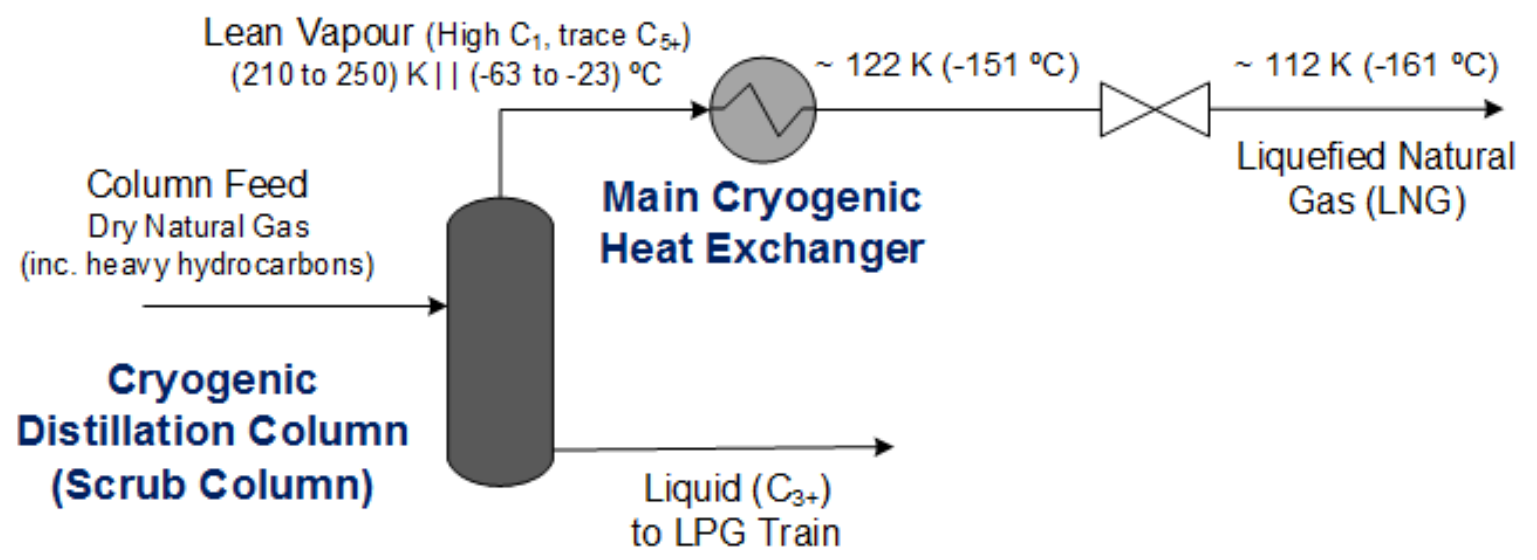

Figure 1: Schematic of a cryogenic distillation column feeding to the main cryogenic heat exchanger in a liquefaction plant with approximate operational temperatures.

The unwanted carry-over of heavy compounds into the vapour overheads that enter the MCHE represents a significant operational problem, particularly for mega-scale LNG plants. This can occur for several reasons including (1) variations in the feed entering the liquefaction plant, (2) the distillation column's operation is not adequate, (3) a process upset occurs upstream of the column or (4) the need to increase the heating value of the LNG through the re-injection of heavier hydrocarbons [9]. Carry-over of heavy compounds could lead to solid freeze-out within the MCHE. Deposition of solids reduces the heat transfer efficiency of the MCHE and leads to increased operational costs. Ultimately, a continued build-up of solids in the MCHE will result in a blockage and the unplanned shutdown of the plant. In a mega-scale LNG train where the consequences of such a shutdown are greatest, removal of the blockage and the subsequent plant re-start will require that the MCHE be taken through a $300 \mathrm{~K}$ temperature cycle. As the MCHE is both massive and sensitive to thermal shocks, any such temperature cycle is likely to take several days or a week and thereby adversely impact the stringent schedule of LNG shipments.

An understanding of the phase behaviour and physical properties of the fluid entering the liquefaction plant is fundamental to preventing solid formation. Most recent efforts to improve LNG process simulations have focussed on the use of complex equations of state capable of more accurately describing the vapour-liquid equilibrium and heat capacities of multi-component fluid mixtures [10-14]; however, solid formation prediction using such models is not straightforward. Simulation tools such as OLGA $^{\mathrm{TM}}$, LedaFlow ${ }^{\mathrm{TM}}$ and HyFAST $[15,16]$, which are capable of describing both the hydrodynamics of multiphase flow and the deposition of solids, are available for flow assurance design in upstream pipelines. However, no equivalent 'flow assurance' tool combining material, energy and momentum balance 
models with predictions of solid solubility in light hydrocarbon systems is available for predicting solid deposition in LNG production or other cryogenic operations. The Gas Processors Association (GPA) KL Solid Solubility Program 1.0 (KLSSP) [17] comes closest. KLSSP contains thermodynamic models for predicting solid solubilities in LNG mixtures based on the extensive experimental work of Kohn, Luks and co-workers [18-29]. However, it cannot predict where solid deposition may occur within the MCHE, and what the associated operational symptoms might be.

This work presents a new simulation tool, CryoFAST, and its associated thermodynamic property package, ThermoFAST, which are designed to help address this knowledge gap and thereby help improve the reliability of LNG production. Figure 2 presents a conceptual diagram that represents what these tools calculate and how they can be applied. In Section 2, the solid solubility models embedded in the ThermoFAST tool are compared with literature data and the predictions of the KLSSP software. In Section 3, the framework and component models used for the material, energy and momentum balances are presented, while in Section 4 the use of CryoFAST is demonstrated through a case study of the cryogenic solids blockage incident that occurred in Train 4 of the RasGas plant as documented by Ismail and Al Thani [30].

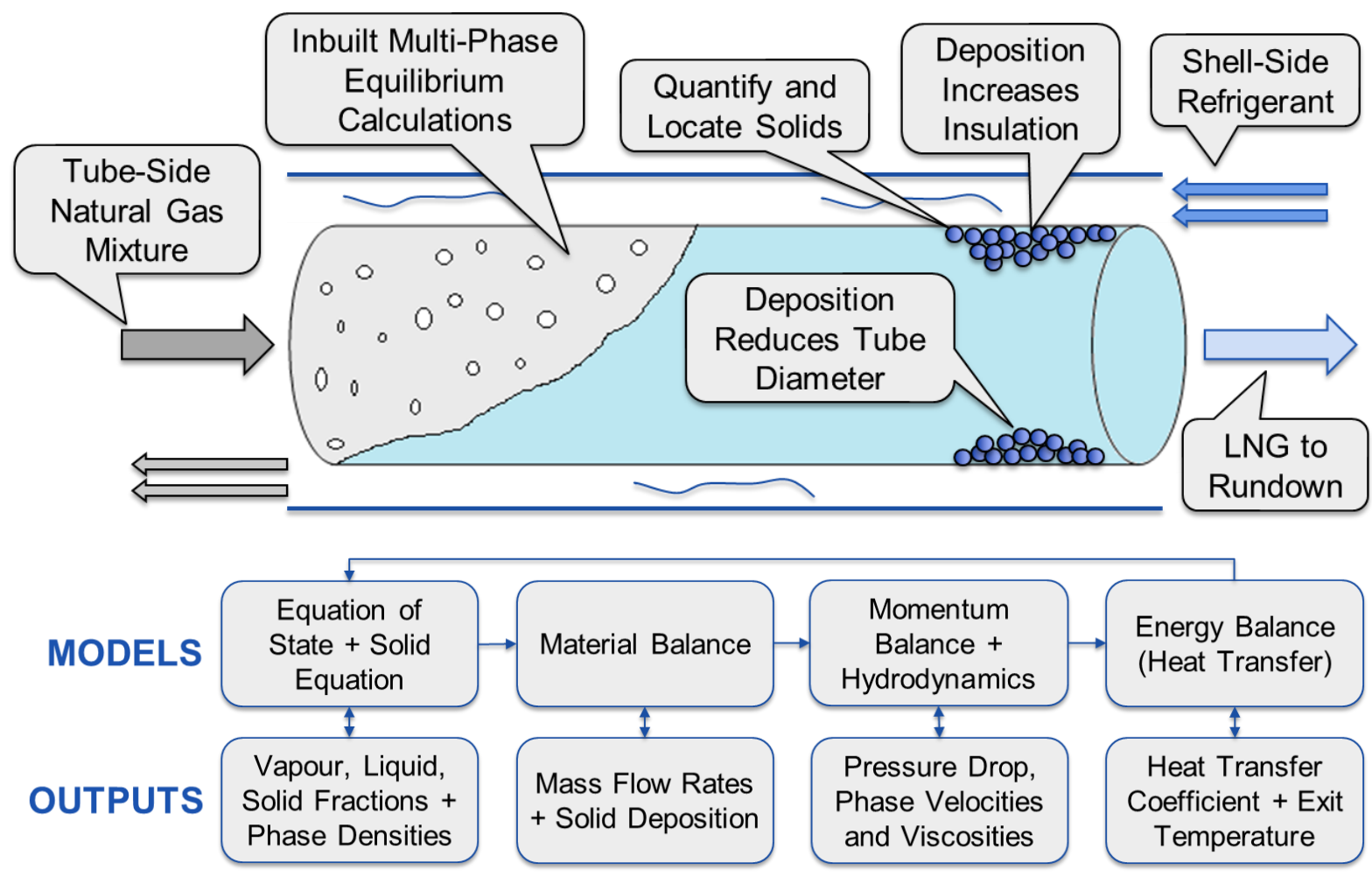

Figure 2: Conceptual diagram of the simulation tool, CryoFAST, described in this work. 


\section{Solid Solubility Calculations}

\subsection{Model Description: ThermoFAST}

ThermoFAST utilises the Peng-Robinson equation of state (PR-EOS) [31] to calculate the thermodynamic properties of multi-component fluid mixtures, including vapour-liquid phase equilibrium, at a specified temperature, $T$, pressure $P$ and overall mole fraction composition, $z_{\mathrm{i}}$. To determine the equilibrium solid solubility, a specialised flash algorithm is followed (Figure 3). The first stage of the algorithm involves calculating the fugacity of pure component $i$ in the solid phase, $f_{i}^{S}$, using an equation first derived by Hildebrand and Scott [32] and endorsed by Prausnitz [33]

$$
\begin{aligned}
\ln \left(f_{i}^{S}\right)= & \ln \left(\varphi_{\text {pure }, i}^{L} P\right)-\frac{\Delta H_{f, i}}{R T_{m, i}}\left[\frac{T_{m, i}}{T}-1\right]+\frac{\Delta c_{p, i}^{L \rightarrow S}}{R}\left[\frac{T_{m, i}}{T}-1+\ln \left(\frac{T}{T_{m, i}}\right)\right] \\
& -\frac{\Delta v_{i}^{L \rightarrow S}\left(P-P_{m}\right)}{R T}
\end{aligned}
$$

Here, $\varphi_{\text {pure }, i}^{L}$ is the fugacity coefficient of pure component $i$ in the liquid phase, which is calculated using the PR EOS; $T_{m, i}$ is the melting temperature of pure component $i ; \Delta H_{f, i}$ is the corresponding molar heat of fusion at $T_{m, i} ; \Delta c_{p, i}^{L \rightarrow S}$ is the molar specific heat of the component as a pure liquid minus that of the pure solid $i$, which is approximated as being independent of temperature; $\Delta v_{i}^{L \rightarrow S}$ is the change in volume between the liquid and solid phase of component $i$; and $P_{m}$ is a reference pressure for the given melting temperature, which in this case is at atmospheric pressure. Each term on the right of eq (1) is calculable from knowledge of the pure component properties, which were taken from various sources including the DIPPR® database [34-37]. The algorithm allows for the possibility that more than one solid phase exists but requires each solid phase to be a pure component. Under these circumstances, the total solid phase fraction is $S_{F}=\Sigma s_{i}$, where $s_{i}$ is the solid phase mole fraction of solute $i$ that is calculated via the material balance relationship,

$$
s_{i}=z_{i}-x_{i} L
$$

where, $L$ is the total liquid phase mole fraction, and $x_{\mathrm{i}}$ is the mole fraction of component $i$ in the liquid phase. At equilibrium, a component's liquid phase mole fraction is related to the fugacity of the solid solute and the partial fugacity coefficient of that component in the liquid phase mixture, $\varphi_{i}^{L}$ :

$$
x_{i}=\frac{f_{i}^{S}}{\varphi_{i}^{L} P}
$$


By initially setting $L=1, S_{F}=0$, and $x_{\mathrm{i}}=z_{\mathrm{i}}$, eqs (2) and (3) can be solved iteratively as shown in Figure 3 until the value of $L$ converges to a numerically stable value. Figure 3 also shows how this algorithm is extended to describe systems at solid-liquid-vapour equilibrium (SLVE) by first performing a VLE flash to calculate the vapour fraction, $\beta$, of the fluid phases evaluating $f_{i}^{s}$ using eq (1), and then iteratively solving the material balance and equilibrium equations (generalisation of eqs (2) and (3)) across all phases present.

SOLID-LIQUID EQUILIBRIUM (SLE FLASH)

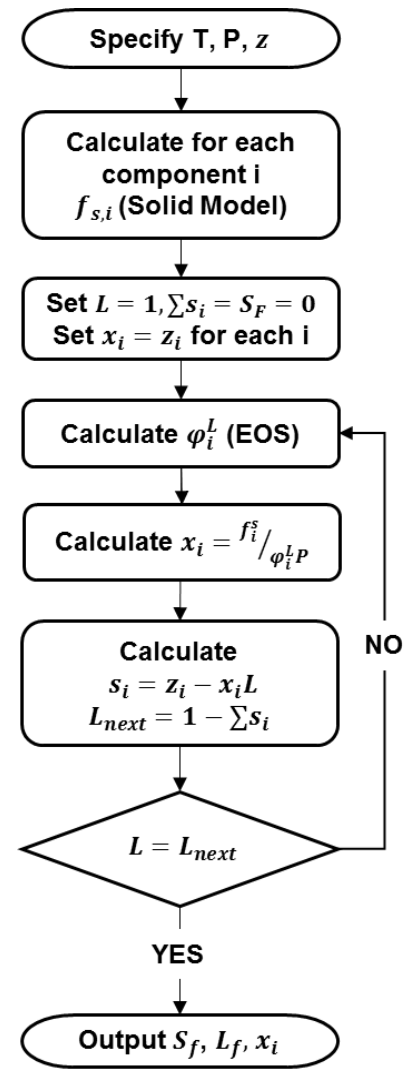

SOLID-LIQUID-VAPOUR MULTIPHASE EQUILIBRIUM (COMBINES SLE + VLE ALGORITHMS)

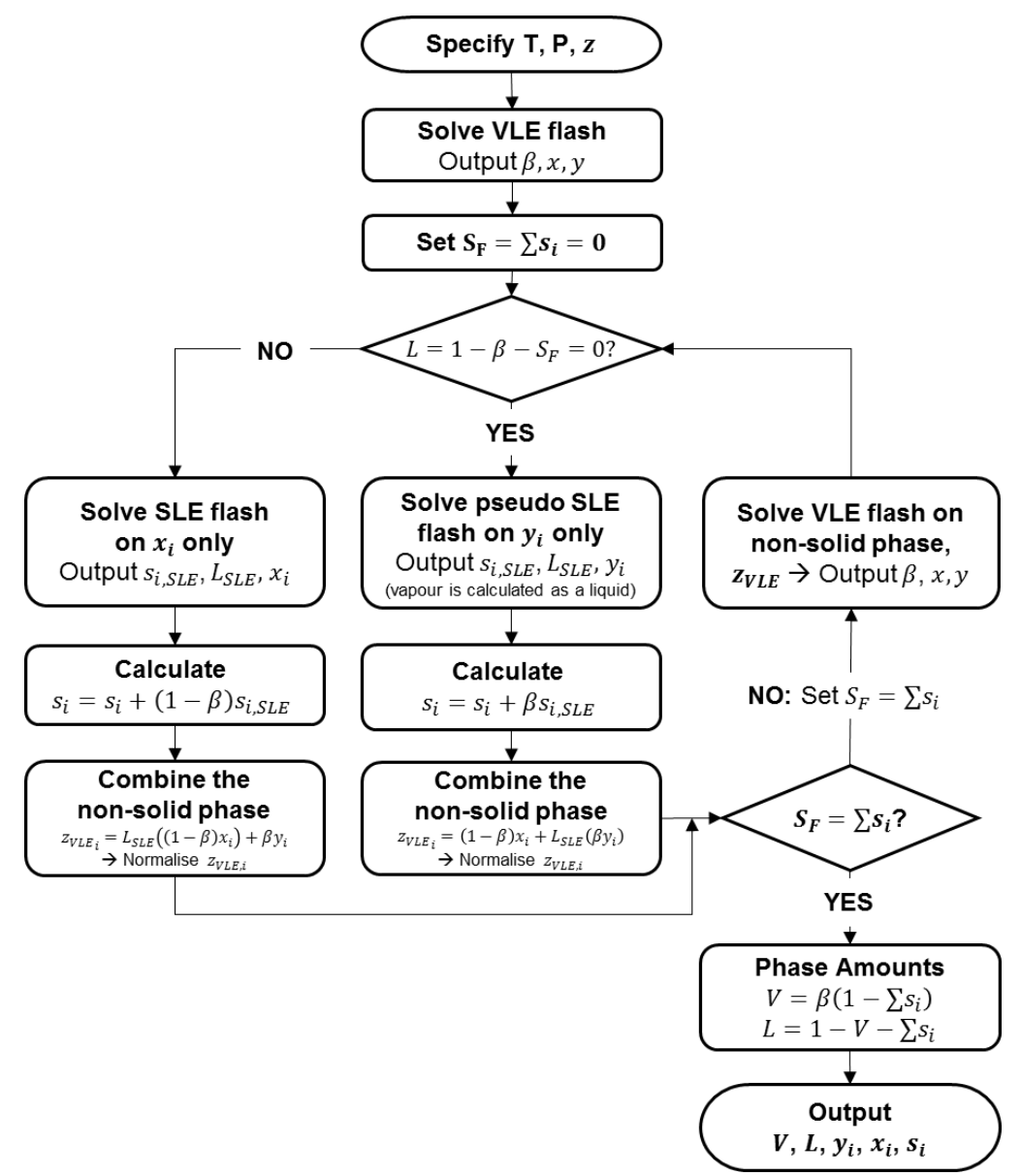

Figure 3: LEFT shows the isothermal solid-liquid equilibrium flash algorithm (where liquid can be substituted for vapour to calculate solid-vapour equilibrium) using both a solid freeze-out model and a cubic EOS for the fluid phase composition. RIGHT shows the isothermal multiphase equilibrium flash algorithm combining an EOS for the vapour-liquid equilibrium (VLE) calculations and the solid solubility equation (SLE Flash). Here $T$ is temperature, $P$ is pressure, $z$ is overall composition, $i$ represents a particular single component, $f$ is fugacity, $s$ is the solid composition, $x$ is liquid composition, $y$ is vapour composition, $\varphi^{L}$ is the partial fugacity in the liquid phase, $\beta$ is the vapour fraction of the non-solid fluid phases, $V$ is the overall vapour mole fraction, $L$ is the overall liquid mole fraction and the subscript SLE refers to any variable determined from the SLE Flash algorithm within the multiphase equilibrium calculation. 


\subsection{Comparisons to Literature Data}

To demonstrate the ability of ThermoFAST to predict reliably the melting temperatures of heavy hydrocarbon solutes in light hydrocarbon solvents, comparisons were made for four exemplar binary hydrocarbon systems with readily available experimental data (Figure 4): (a) methane + benzene [38], (b) ethane + benzene [20], (c) methane + hexane [38-41] and (d) methane + toluene [38]. Each of the considered heavy hydrocarbon solutes poses high risk of freeze-out within the MCHE. Comparisons were also made with KLSSP, which employs a distinctly different thermodynamic model where the activity coefficient of the solute in the liquid mixture is described by an empirical function of temperature and composition; the KLSSP model contains no pressure dependence and is valid over a relatively limited range of composition [17].

The measurements for each system were acquired at pressures from (0.1 to 13.7) $\mathrm{MPa}$, (0.07 to 0.99) $\mathrm{MPa},(0.3$ to 13.2$) \mathrm{MPa}$ and (5.8 to 13.4) MPa, respectively. ThermoFAST is able to capture the pressure dependence of the data across the entire composition range, with a root mean square (RMS) deviation of $1.8 \mathrm{~K}, 1.0 \mathrm{~K}, 0.9 \mathrm{~K}$ and $1.6 \mathrm{~K}$, respectively. In contrast, KLSSP is unable to capture the pressure dependence of the data and can diverge significantly from the measurements. Even when some significant outliers are excluded, the RMS deviation between the data and the predictions of the KLSSP model are $8.2 \mathrm{~K}$ for the methane + benzene system, $5.6 \mathrm{~K}$ for the ethane + benzene system and $3.7 \mathrm{~K}$ for the methane + hexane system.

No comparisons with KLSSP could be made for the methane + toluene data set because the model is unable to perform calculations for systems containing toluene. Additionally, we note that the standard interface of the KLSSP software package places limits on the allowable specification of concentration for various components: for hexane and benzene, these are less than 0.2 and 0.001 mole fraction, respectively. Consequently, only $58 \%, 92 \%$ and $0 \%$ of the data comparisons for methane + hexane, methane + benzene and ethane + benzene systems, respectively, could be calculated using the standard interface of the KLSSP software. Comparisons for the remaining conditions shown required a by-pass of the standard interface by utilising the internal Visual Basic for Applications interface implemented in Microsoft Excel. 

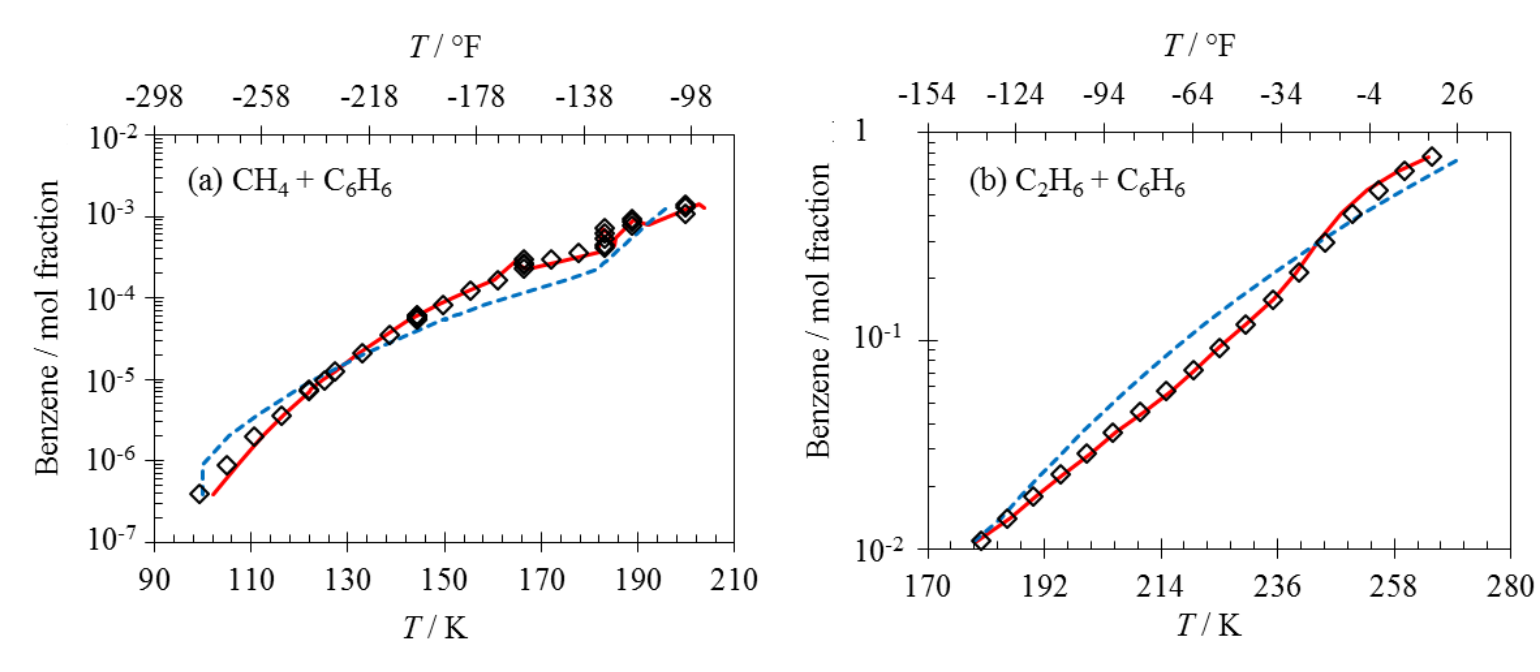

$\diamond \quad$ Kuebler \& McKinley (1974) — ThermoFAST ----- KLSSP
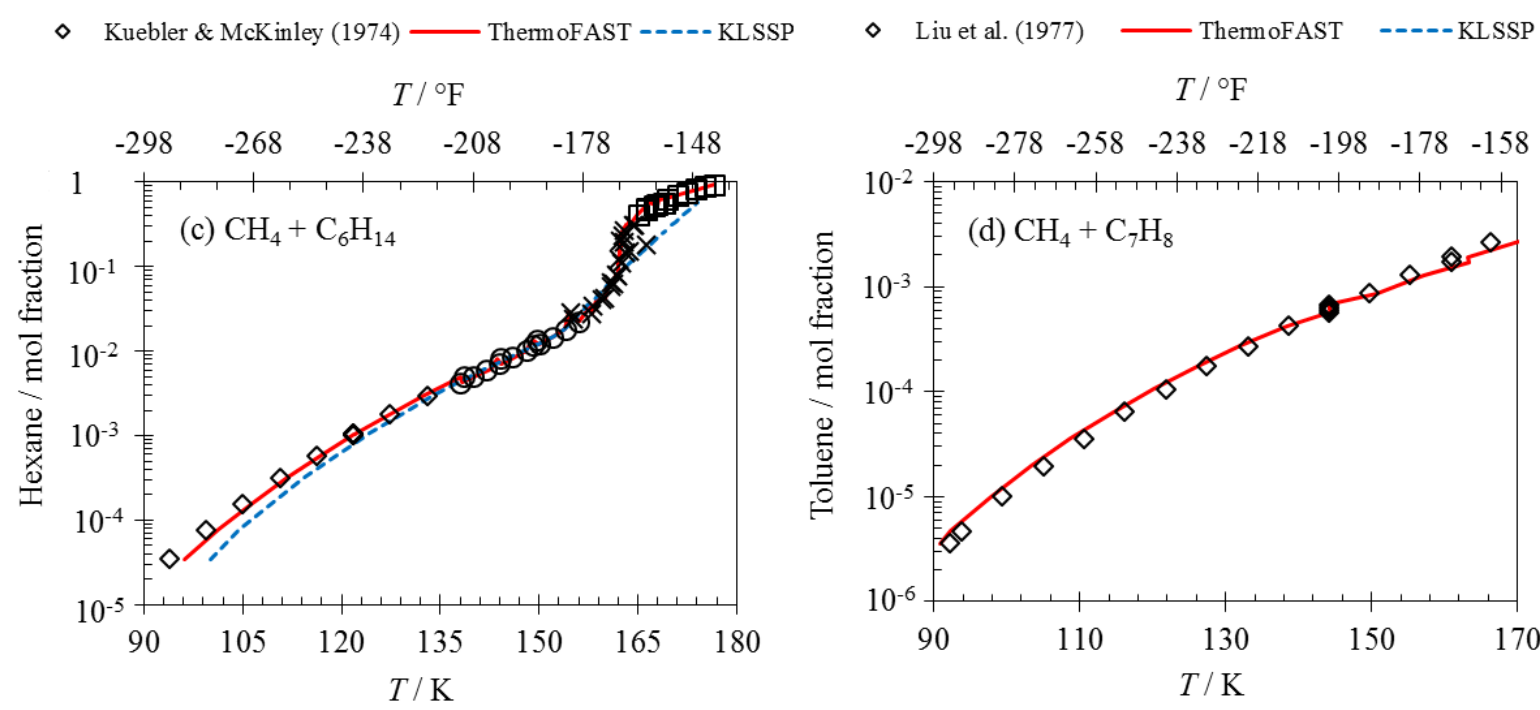

$\diamond$ Shim \& Kohn (1962) O Dickinson et al. (1973)

$\times \quad$ Kuebler \& McKinley (1974) $\square$ Luks et al. (1981)

$\diamond$ Kuebler \& McKinley (1974) — ThermoFAST

Figure 4: Solubility curves for (a) methane + benzene, (b) ethane + benzene, (c) methane + hexane and (d) methane + toluene, comparing ThermoFAST solid solubility predictions against experimental data [20,38-41] and KLSSP with respect to the absolute temperature (abscissa).

\section{CryoFAST Model}

The structure of the algorithm used by the CryoFAST simulation tool is outlined in Figure 5. The flow path to be simulated is discretised into nodes, each representing an open, constant volume system, which can be solved under steady-state or transient conditions. Solids formation can be calculated for both options; however, deposition implicitly requires a transient (time dependent) solution. The inlet boundary condition is specified to be a constant fluid mixture composition at a fixed temperature and pressure at a constant mass flow rate. The multiphase equilibrium calculations are performed with ThermoFAST at each node to determine the flow rates and densities of the vapour, liquid and solid phases, as shown in step (1) of Figure 5. 


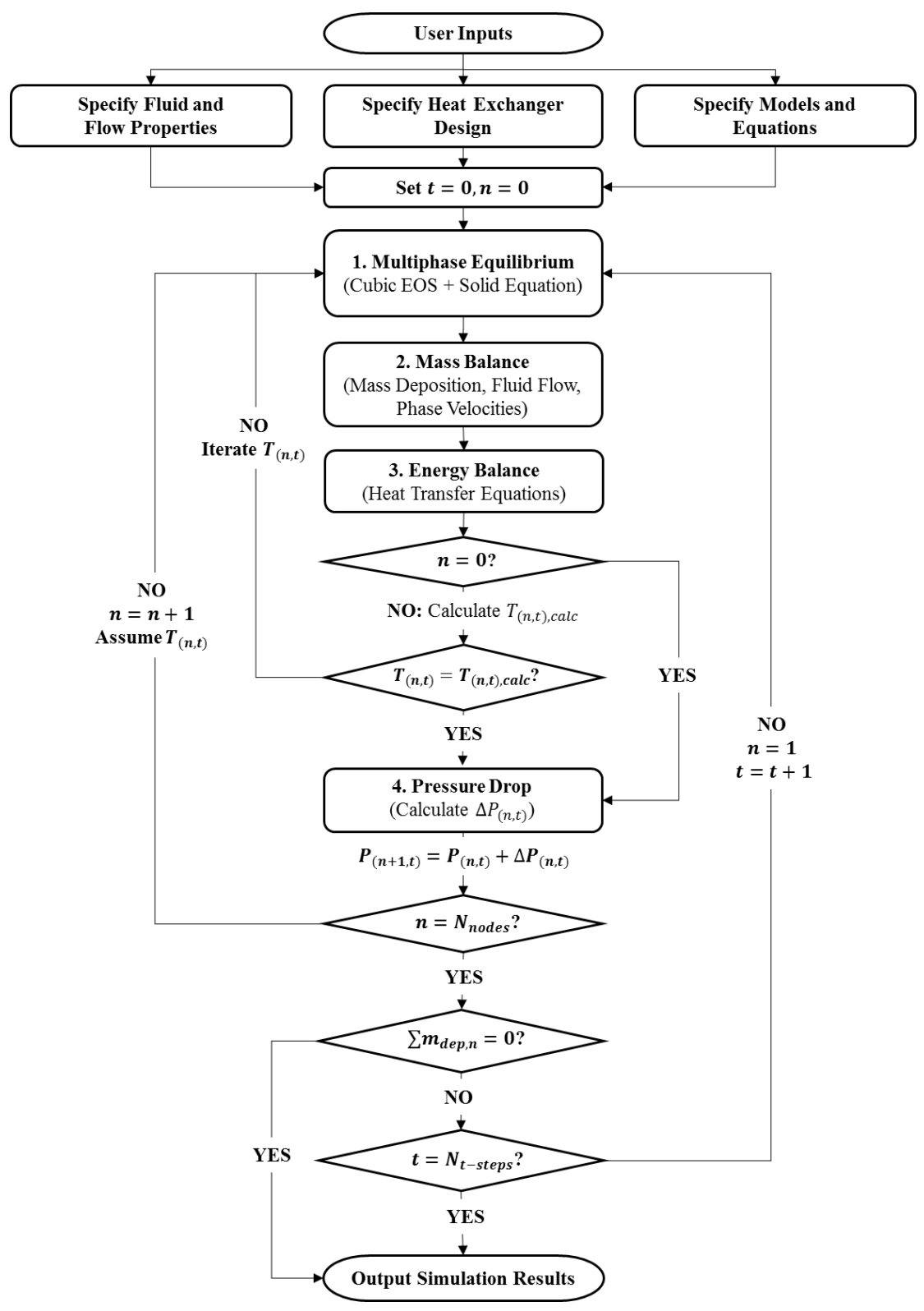

Figure 5: A simplified version of the CryoFAST algorithm for the prediction of solid deposition through a single tube of a cryogenic heat exchanger, where $n$ is the node position, $t$ is the time-step associated with transient behaviour, $T$ is temperature, $P$ is pressure, $m_{\text {dep,t }}$ is total mass deposited during that time-step, $N_{\text {nodes }}$ is total number of nodes and $N_{\text {t-steps }}$ is the total number of time steps (other details explained in text). Following the input of the required specifications by the user the algorithm begins by calculating the vapour, liquid and solid phase fractions and densities utilising ThermoFAST (step 1). Subsequently, material balance in the node is solved to determine the change in mass flow rate and pipe diameter due to potential deposition (step 2). Thereafter, an energy balance (step 3 ) between the fluid and the refrigerant is performed and the temperature of the current node is iterated on until convergence (except for $n=0$ where the temperature is specified). In the final major step (4), the pressure drop is calculated to determine the pressure in the subsequent node. Once mass deposition is zero across all nodes or the total number of time steps defined by the user is reached, the simulation is completed. 


\subsection{Material and Energy Balances}

Solution of the material and energy balances is crucial to any dynamic simulation flow tool and this is done in step (2) and (3) of the CryoFAST algorithm (Figure 5). The transient method differs from traditional time-dependent simulations in that it adopts a simplified approach to reduce computational time. Traditional transient simulations are computationally intensive because they solve the dynamic material and energy balances across the entire system at every step in time. A less accurate but simpler and faster approach to solving this transient problem is to consider a single constant-volume fluid element, and track its evolution as it propagates along the flow path. The rate at which the fluid element propagates along the discretised flow path is determined by calculating a residence time $\Delta t$ for each node as shown in Figure 6.

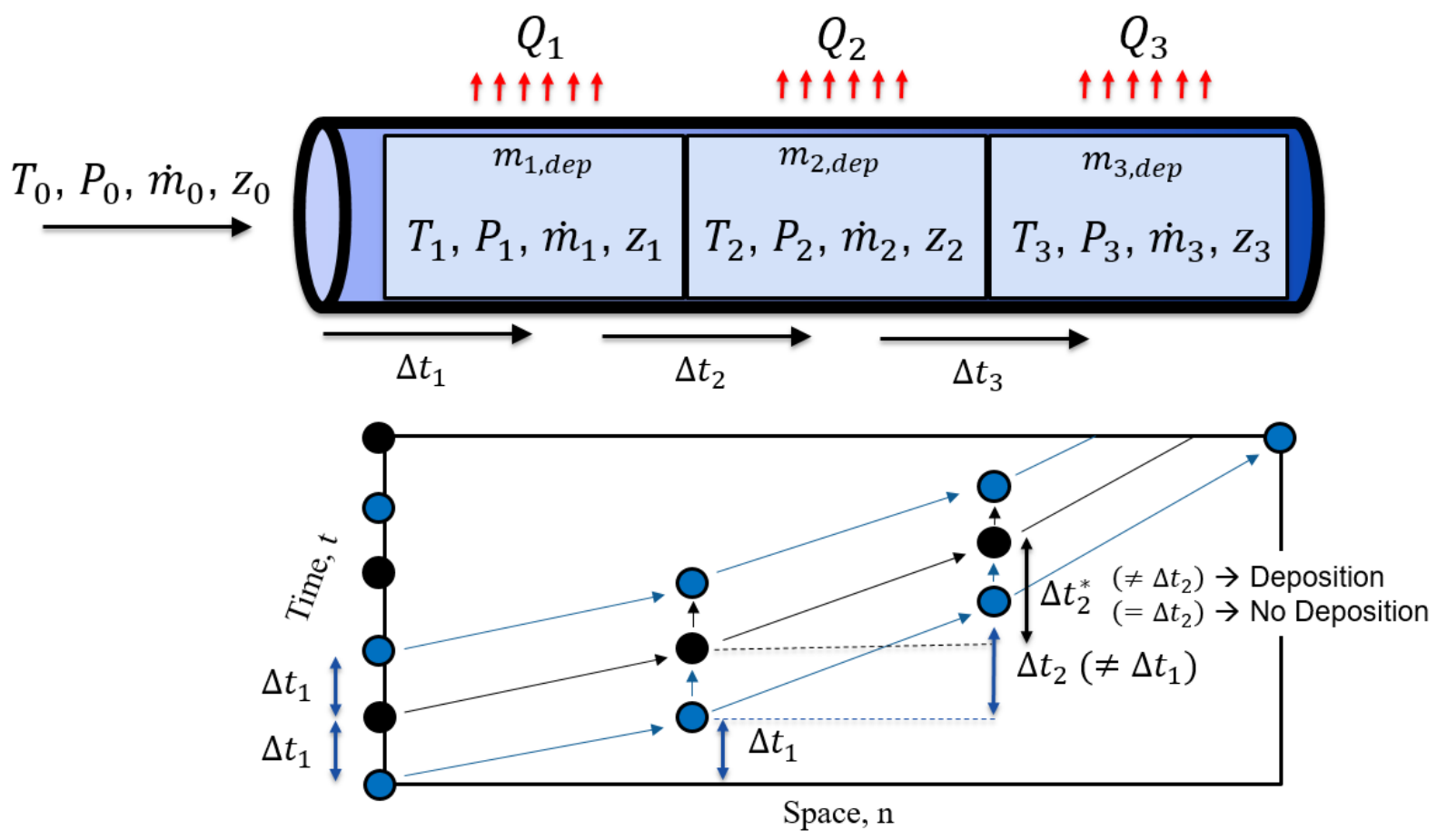

Figure 6: Simplified, rapid method for solving transient simulations. The evolution of a single constant volume fluid package propagating along the first three nodes within the system across three time steps. Here $T$ is temperature, $P$ is pressure, $\dot{m}$ is mass flow rate, $z$ is the overall composition, $Q$ is the heat transferred to the environment, $\Delta t$ is the time step and $n$ the node number.

The residence time at the node is related to the node spacing, $L_{n}$, and the fluid velocity inside the node $u_{\mathrm{n}}$

$$
\Delta t=L_{n} / u_{n}
$$

The velocity of the fluid element is dependent upon mass flow rate $\dot{m}$ and the mass density of the fluid at that node $\rho_{n}$ (which depends on the local $T, P$, and $z$ ) according to, 


$$
u_{n}=\frac{\dot{m}}{\rho_{n} V}
$$

where $V$ is the volume of the node, or equivalently, the fluid element (equal to $L$ times the pipe's cross-sectional area). In the absence of deposition, the mass flow rate remains constant as required by material balance. The absolute mass of the constant volume fluid element generally increases as it cools in the cryogenic heat exchanger and the fluid's density increases.

For a given $(T, P, z)$, the ThermoFAST algorithm determines the solid phase fraction present in the node. If solids are predicted to form, then some or all of the solids are deposited in the node and any remaining solids are assumed to exist as a suspended dispersion within the continuous fluid phase. A detailed hydrodynamic calculation involving consideration of, for example, the adhesive and cohesive forces between solid particles and the pipe wall as well as the shear forces exerted on any solid aggregates or deposits would be required to predict the physical extent of deposition versus dispersion. Instead, the user simply specifies a deposition fraction, $\chi$, between 0 (no deposition) and 1 (complete deposition) for the material balance calculations:

$$
\begin{aligned}
& \chi S_{F} \rho_{n} V=m_{\text {dep }}=\left(\dot{m}_{\text {in }}-\dot{m}_{\text {out }}\right) \Delta t \\
& m_{\text {dep }, i}=\left(\dot{m}_{\text {in }} w_{i, \text { in }}-\dot{m}_{\text {out }} w_{i, \text { out }}\right) \Delta t
\end{aligned}
$$

Here $\dot{m}_{\text {in }}$ is the mass flow rate entering a node, $\dot{m}_{\text {out }}$ is the mass flow rate leaving the node, $m_{d e p, i}$ is the solid mass deposited in the node for component $i$ and $w_{i, \text { in }}$ and $w_{i, o u t}$ represent the mass fractions of component $i$ entering and leaving the node, respectively. Eqs (6) and (7) are used to solve for the unknowns $\dot{m}_{\text {out }}$ and $w_{i, \text { out }}$, all other quantities either being specified (by the user and/or the previous node's effluent) or calculated with the ThermoFAST model. A steady-state solution for a given node corresponds to either $S_{\mathrm{F}}=0$ (no solids present) or $\chi=0$ (no solids deposited), in which case the inlet and outlet quantities are equal and the time-step becomes arbitrary.

Following the material balance calculation, CryoFAST evaluates an energy balance in step (3) using the following equation for an open system,

$$
\begin{aligned}
\frac{1}{\Delta t} \sum m_{\text {dep }, i} \Delta & h_{f u s, i} \\
& =\dot{m}_{\text {in }}\left(h_{\text {in }}+\frac{u_{\text {in }}^{2}}{2}+g z_{H, \text { in }}\right)-\dot{m}_{\text {out }}\left(h_{\text {out }}+\frac{u_{\text {out }}^{2}}{2}+g z_{H, \text { out }}\right)+\dot{Q}
\end{aligned}
$$


where, $\Delta h_{f u s, i}$ is the specific heat of fusion of each solid component deposited, $h$ is the specific enthalpy of the fluid flowing between the inlet (in) and the outlet (out) of the node including vapour, liquid and solid contributions, $g$ is the acceleration due to gravity, $z_{H}$ is the vertical height, $\dot{Q}$ is the rate of heat transfer to the environment and $u$ is the velocity of the (multiphase) fluid. The last quantity, $u$, refers to the constant volume fluid element propagating through each node within the heat exchanger, which varies as a result of hydrodynamic effects and changes to fluid density, as per eq (5).

The velocity of the multiphase fluid, $u$, is used to calculate the vapour and liquid phase velocities that are required in the calculation of pressure drop. The specific phase velocities are determined through a drift flux relationship model by first utilising the correlation by Nicklin et al. (1962) [42],

$$
u_{V}=C_{D} u+u_{D}
$$

where $u_{D}$ is the drift velocity in a stagnant fluid, $C_{D}$ is the distribution factor and $u_{V}$ is the vapour phase velocity. The values of $u_{D}$ and $C_{D}$ are estimated with a correlation by Bendiksen (1984) [43] that considers the Froude number $\left(F r_{N}\right)$ to quantify the gravitational and inertial forces.

$$
\begin{gathered}
F r_{N}=\frac{u}{\sqrt{g D}} \\
\left\{\begin{array}{llc}
\text { If } F r_{N}>F r_{\text {crit }}=3.5, & C_{D}=1.2, & u_{D}=0 \\
\text { If } F r_{N} \leq F r_{\text {crit }}=3.5, & C_{D}=1.05 & u_{D}=0.54 \sqrt{g D}
\end{array}\right\}
\end{gathered}
$$

Here $D$ is the diameter of the tube. The superficial velocities for both the vapour, $u_{S V}$, and liquid, $u_{S L}$, are calculated from the vapour phase volume fraction, $\phi_{V}$, using

$$
\begin{gathered}
u_{S V}=\phi_{V} u_{V} \\
u_{S L}=u-u_{S V} \\
u_{L}=\frac{u_{S L}}{1-\phi_{V}}
\end{gathered}
$$

The rate of heat transfer to the environment is calculated from the external surface area of the node volume, $A$, a heat transfer coefficient, $U$, and $\log$ mean temperature difference for counter current flow, $\Delta T_{L M T D}[44]$,

$$
\dot{Q}=U A \Delta T_{L M T D}
$$


To determine $\Delta T_{L M T D}$ the CryoFAST algorithm requires the user to specify the temperature of the environment (or refrigerant) at the position of each node. At nodes where deposition occurs, the local heat transfer becomes $(U A)_{d e p}$ and is calculated using a method given by Haj-Shafiei et al. (2014) [45],

$$
\frac{1}{(U A)_{d e p}}=\frac{1}{U_{N D} A}+\left[\frac{\ln \left(\frac{R}{R-\delta}\right)}{2 \pi k_{d e p} L}\right]
$$

Here, $U_{N D}$ is the overall heat transfer coefficient in the absence of deposition (a user specified variable), $R$ is the tube's radius, $k_{d e p}$ is the thermal conductivity of the deposit layer, $\delta$ is the radial thickness of the deposit (calculated from $A, m_{\mathrm{dep}}$, and the density of the solid phase assuming zero porosity). Both the thermal conductivity and solid density are estimated from the DIPPR ${ }^{\mathrm{TM}} 801$ Database (equation \#100) [46].

Except for the inlet boundary (node 0), solution of the material and energy balances in eqs (5) - (8) requires an initial assumption of the node's temperature to determine $m_{\mathrm{dep}}, s_{\mathrm{i}}, \dot{m}_{\text {out }}$ and $w_{i, o u t}$ before eq (8) is solved for $h_{\text {out }}$, which in turn provides a new estimate for the node's temperature from the PR EOS and the node pressure. The calculations are repeated iteratively until the estimated node temperature matches that determined from eq (8) within a tolerance of $10^{-3} \mathrm{~K}$. The pressure drop across the node is then calculated to enable the pressure of the next node to be determined.

\subsection{Pressure Drop: Centrifugal and Twisting Effects}

The final major step in the algorithm (step (4) of Figure 5) involves the calculation of the pressure drop through the tube, for which CryoFAST utilises the 1973 correlation of Beggs and Brill [47]. This correlation is capable of handling both horizontal and vertical flow for multiphase fluids and uses changes in the fluid phase velocities, densities and viscosities across the node to determine the resulting pressure drop. In principle, an iterative calculation for the pressure in a node should be conducted to account fully for the impact of the hydrodynamic pressure drop on the thermodynamic properties of the fluid element at that node. However, if a sufficient number of nodes (e.g. 200 as discussed below) are used in the simulation, the change in the calculated properties that results upon implementation of the full iterative solution is negligible, with little impact on the final result despite significantly increasing the computational load. Thus, in the current implementation of the CryoFAST 
model, the pressure drop calculated using the Beggs and Brill [47] is simply applied to the subsequent node.

The orientation and type of heat exchanger plays a significant role in the MCHE affecting in principle both the pressure drop and velocity through variation of the flow regime; certain geometries can even create stagnation zones. For simplicity it is assumed in the CryoFAST model that geometry only affects pressure drop and has negligible effect on velocity changes due to variations in flow regime.

However, centrifugal and twisting effects must be considered to describe reliably the pressure drop that occurs in one of the two most common types of MCHE used in liquefaction plants: coil (or spiral) wound heat exchangers (CWHE) [48]. The CryoFAST software package allows the user to specify whether the heat exchanger being considered is a CWHE, in which case the user must specify the radius of the bundle, $R_{c}$, and coil pitch, $\Lambda_{c}$, as shown in Figure 7.

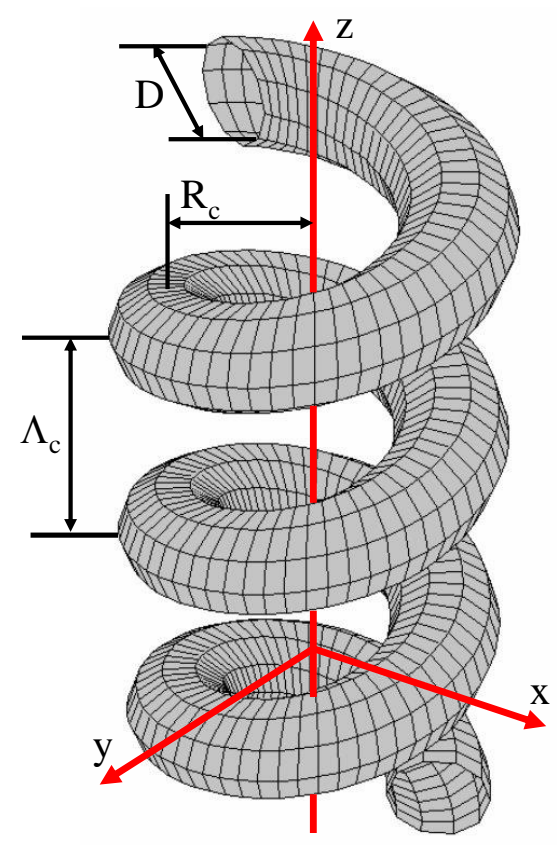

Figure 7: Diagram of a coil showing the diameter of the tube, $D$, the coil's pitch, $\Lambda_{c}$, and radius of the coil bundle, $R_{C}$ [49].

Multiple studies [50-53] have shown the effect that both centrifugal and twisting forces can have on flow through helical tubes. Both these forces can be quantified respectively by the Dean number $(D e)$ and Germano number $(G e)[49]$ :

$$
D e=\operatorname{Re} \sqrt{\kappa}
$$




$$
G e=\operatorname{Re} \tau
$$

where, $R e$ is the Reynolds number and $\kappa$ and $\tau$ are given by the following expressions:

$$
\begin{aligned}
& \kappa=\frac{R_{c} / R}{\left(R_{c} / R\right)^{2}+\left(\Lambda_{c} / 2 \pi R\right)^{2}} \\
& \tau=\frac{\Lambda_{c} / 2 \pi R}{\left(R_{c} / R\right)^{2}+\left(\Lambda_{c} / 2 \pi R\right)^{2}}
\end{aligned}
$$

These two dimensionless groups are used to calculate the relative friction factor multiplier, $f_{R}$, which increases the effective friction factor, $f$, required in the Beggs and Brill correlation for pressure drop.

$$
f_{R}=1+0.0167 \frac{D e^{1.5}}{G e}
$$

The other contribution to the effective friction factor used in the Beggs and Brill correlation is Darcy-Weisbach friction factor, $f_{D W}$, for turbulent flow which is calculated by solving the implicit equation developed by Colebrook and White (1937) [54],

$$
\frac{1}{\sqrt{f_{D W}}}=-2 \log \left(\frac{\varepsilon}{3.7 D}+\frac{2.51}{R e \sqrt{f_{D W}}}\right)
$$

Here, $\varepsilon$ is the height roughness of the pipe, $D_{h}$ is the tube's diameter and $R e$ is the Reynolds number. The effective friction factor for coil wound heat exchangers used in the Beggs and Brill correlation for pressure drop is then,

$$
f=f_{R} f_{D W}
$$

\section{Case Study Results \& Discussion}

\subsection{RasGas Train 4 Operating Data}

Ismail and $\mathrm{Al}$ Thani reported plant data for LNG Train 4 in the RasGas complex, resulting in a MCHE blockage and forcing an unplanned shutdown in 2014 [30]. Figure 8 shows operating data from Train 4 for a normal operating period (lasting many months) prior to a scheduled shutdown in August, and from the subsequent start-up until an unplanned 
shutdown in December (114 days later) was forced by the excessive pressure drop across the MCHE.
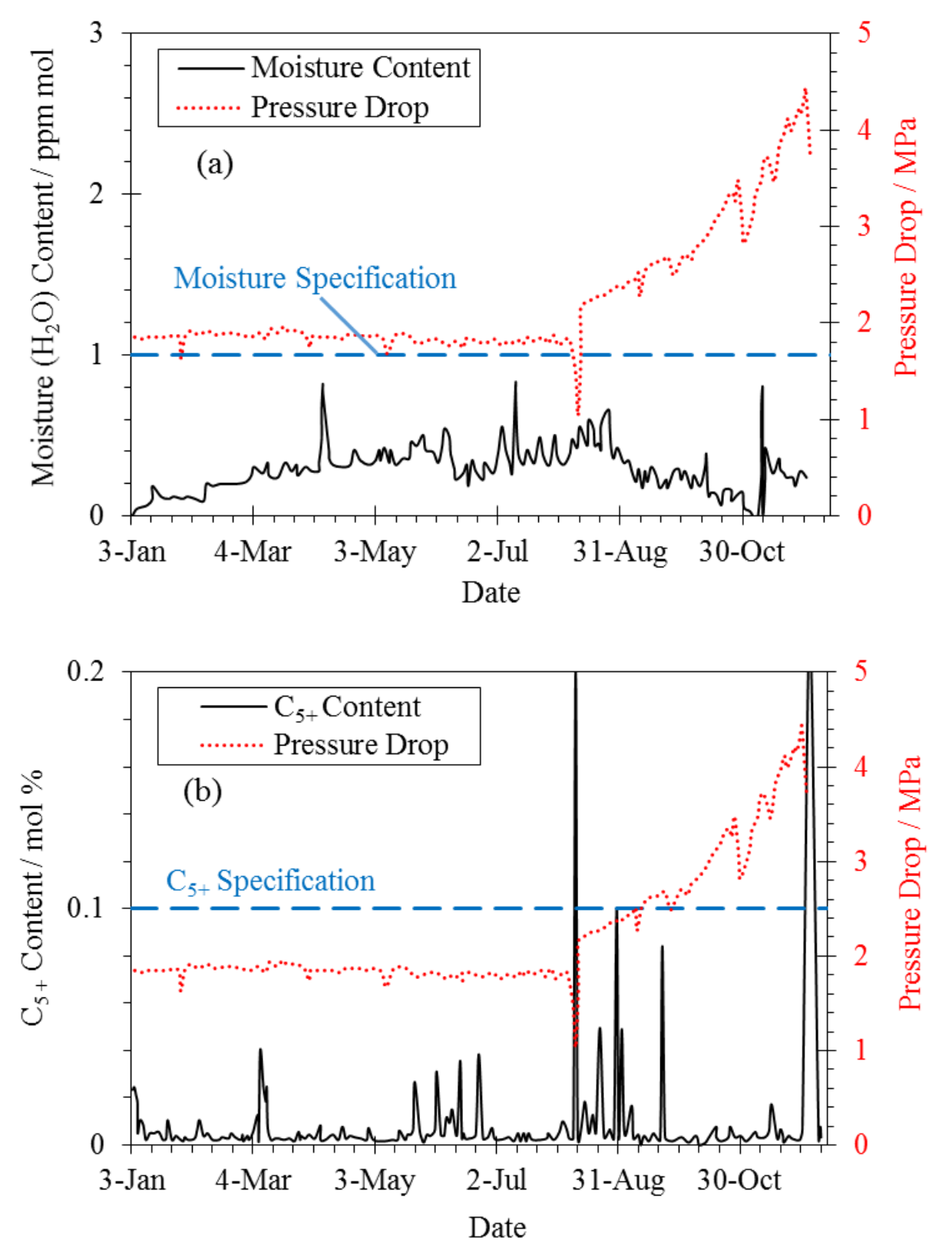

Figure 8: Operating data that has been adapted from RasGas LNG Train 4 in 2014 based on the data reported by Ismail and Al Thani [30] showing (a) the moisture and (b) the $\mathrm{C}_{5+}$ content entering the MCHE. Normal operation was sustained until a scheduled shut-down in August. Following re-start the MCHE pressure drop increased, forcing an unplanned shutdown in December. 
The subsequent analysis reported by Ismail and Al Thani [30] identified the blockage's root cause to be a total loss of reflux flow in the scrub column for 50 seconds during the startup. This was so brief that it was judged by the start-up team to be inconsequential, even though it led to a temporary increase in the $\mathrm{C}_{5+}$ fraction entering the heat exchanger to about $0.21 \mathrm{~mol} \%$, more than double the specification. Over the next two months, the sustained increase in pressure drop was not considered concerning due to other changes in operating condition driven by external factors. The presence of a blockage was unambiguously identified from the operating long-term data and remedial actions were attempted in November 2014. However, by this time a shut-down proved unavoidable and this occurred in December 2014.

Here we demonstrate how application of the CryoFAST simulation tool would have enabled prediction of the location of the heavy hydrocarbon freeze out in the MCHE and the assessment of possible remedial actions at a much earlier stage. Additionally, analysis of the incident using CryoFAST provides some insight into the time frame available following the initial freeze out in which remedial action would likely have been most effective.

\subsection{Simulating the Normal and Upset Operating Conditions}

The feed compositions used for the CryoFAST simulation of the RasGas LNG Train 4 normal and upset operating conditions are shown in Table 1. These compositions were estimated from a combination of the plant data reported by Ismail and Al Thani [30] and a feed composition reported by Al-Muhammadi et al. for the Qatar/RasGas LNG plant [55], where the latter provided the ratio of the $\mathrm{C}_{1}$ to $\mathrm{C}_{4}$ hydrocarbons to the aromatic compounds. For simplicity, the aromatic content of the gas was assumed to be entirely benzene.

Several assumptions were made to enable the necessary specification of geometry for the RasGas LNG Train 4 MCHE. The input parameters for the CWHE are detailed in 
Table 2 for a total simulation time of 50 seconds, it was found that 200 nodes and 10 timesteps were sufficient to achieve convergence (i.e. the simulated results remained constant within $0.1 \%$ with further increases in the number of nodes or time-steps). The heat exchanger design and tube parameters were estimated based on multiple sources including a patent by Truong et al. [56] and other industry literature [57]. The feed mass flow rate was assumed to be the reported maximum capacity of $\sim 4.7$ million tonnes per annum [58] and the number of tubes were chosen to ensure the resulting vapour and liquid velocity within each tube was approximately equal to or less than $10 \mathrm{~m} \cdot \mathrm{s}^{-1}$ and $3 \mathrm{~m} \cdot \mathrm{s}^{-1}$, respectively [44]. The coil pitch is based on an assumed inclination of $10^{\circ}$, and the average of the typical coil diameter of was set to be $1 \mathrm{~m}$ based on the publication of Neeraas [59]. Overall local heat transfer coefficients for a CWHE were reported by Sinnott et al. [44] to be in the range of (900 and $3,500) \mathrm{W} \cdot \mathrm{m}^{-2} \cdot \mathrm{K}^{-1}$, which is considerably higher than the standard shell and tube heat exchanger; a constant value of $900 \mathrm{~W} \cdot \mathrm{m}^{-2} \cdot \mathrm{K}^{-1}$ was chosen for this work. The refrigerant temperature profile was chosen to achieve a temperature approach of $10 \mathrm{~K}$ and a steady state effluent temperature of $122 \mathrm{~K}\left(-151^{\circ} \mathrm{C}\right)$ (prior to the end-flash valve) under the assumption of counter-current flow. Finally, given that no data regarding deposition rates within MCHEs are available within the open literature, any solids formed were assumed to deposit instantly i.e. $\chi=1$ in eq. (6).

Table 1: The estimated dry mole fraction feed compositions for the normal and upset operating simulated with CryoFAST in RasGas LNG Train 4. The basis for these compositions is described in text.

\begin{tabular}{lcc} 
& $\begin{array}{c}\text { Normal Operating } \\
\text { Condition }\end{array}$ & $\begin{array}{c}\text { Upset Condition } \\
\left(\boldsymbol{C}_{5+} \text { Carry Over }\right)\end{array}$ \\
\hline $\mathrm{CO}_{2}$ & 0.000040 & 0.000040 \\
$\mathrm{CH}_{4}$ & 0.909329 & 0.907510 \\
$\mathrm{C}_{2} \mathrm{H}_{6}$ & 0.057492 & 0.057377 \\
$\mathrm{C}_{3} \mathrm{H}_{8}$ & 0.021953 & 0.021910 \\
$\mathrm{iC}_{4} \mathrm{H}_{10}$ & 0.004239 & 0.004230 \\
$\mathrm{nC}_{4} \mathrm{H}_{10}$ & 0.006847 & 0.006833 \\
$\mathrm{iC}_{5} \mathrm{H}_{12}$ & 0.000028 & 0.000596 \\
$\mathrm{nC}_{5} \mathrm{H}_{12}$ & 0.000026 & 0.000764 \\
$\mathrm{C}_{6} \mathrm{H}_{14}$ & 0.000026 & 0.000441 \\
$\mathrm{C}_{7} \mathrm{H}_{16}$ & 0.000016 & 0.000220 \\
$\mathrm{Benzene}_{2}$ & 0.000004 & 0.000078
\end{tabular}


Table 2: Parameters used for the RasGas LNG Train 4 simulations.

\begin{tabular}{|c|c|}
\hline User Input & Value \\
\hline Number of Nodes & 200 \\
\hline Total Simulation Time / s & 50 \\
\hline Number of time-steps & 10 \\
\hline Solid Deposition Fraction /\% & 100 \\
\hline Feed Mass Flow Rate $/ \mathrm{kg} \cdot \mathrm{s}^{-1}$ & 145.87 \\
\hline Feed Temperature / $K$ & 260 \\
\hline Feed Pressure / $M P a$ & 5.0 \\
\hline Wall Roughness / mm & 0.001 \\
\hline Tube Length / $m$ & 100 \\
\hline Number of Tubes & 1,570 \\
\hline Internal Diameter / $\mathrm{mm}$ & 14.8 \\
\hline Average Coil Diameter $/ \mathrm{m}$ & 1 \\
\hline Coil Pitch $/ \mathrm{m}$ & 0.554 \\
\hline Tube Inclination / degrees & 10 \\
\hline Overall Local Heat Transfer Coefficient $/ W \cdot m^{-2} \cdot K^{-1}$ & 900 \\
\hline Refrigerant Temperature (Profile Range) / K & 112 to 250 \\
\hline
\end{tabular}



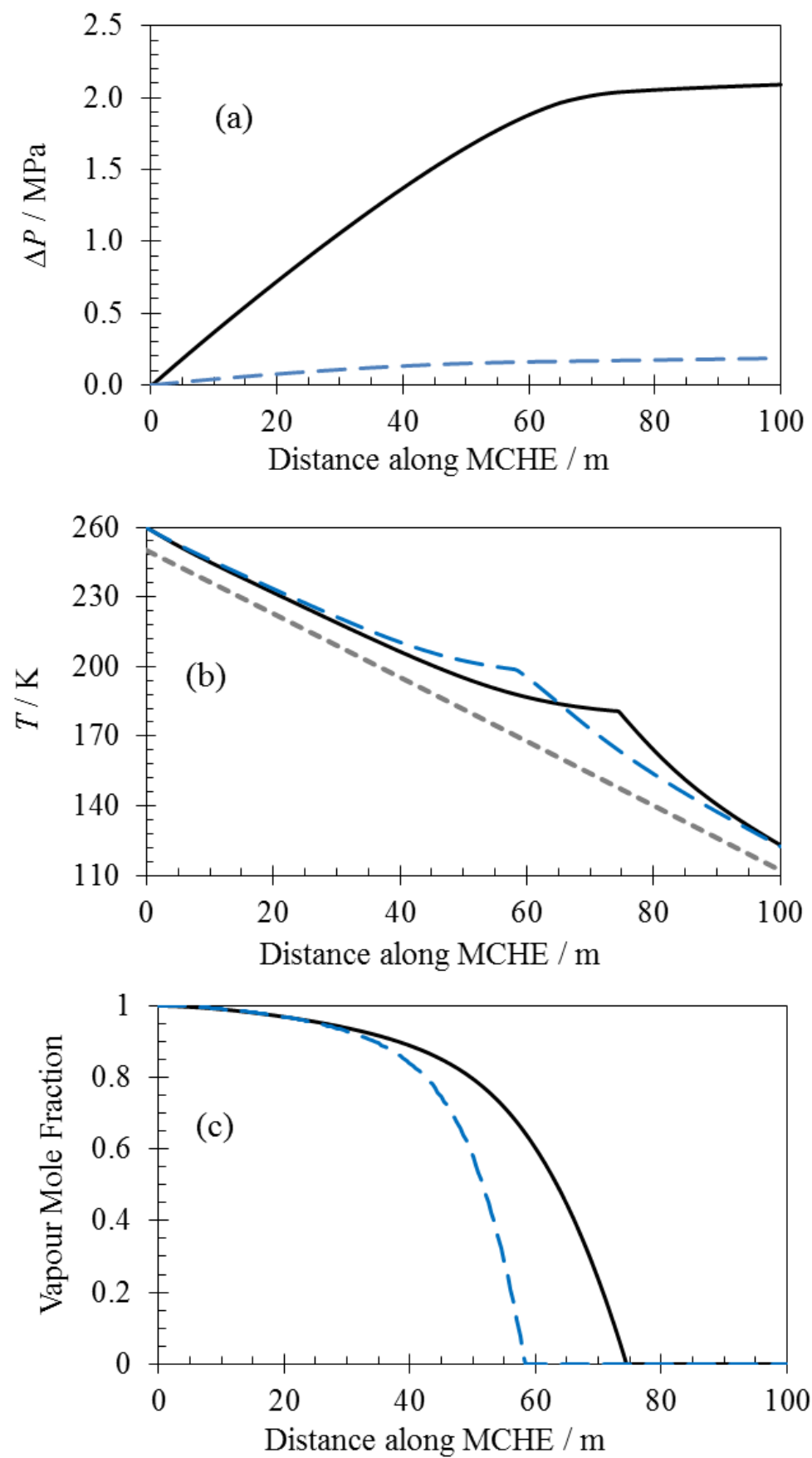

— Full Simulation - - Centrifugal Forces Off -----Refrigerant

Figure 9: Simulation results for RasGas LNG Train 4 under normal operating conditions for (a) pressure drop, $\Delta P$, (b) temperature and (c) vapour mole fraction with distance along the $\mathrm{MCHE}$. Considering centrifugal and twisting forces in the calculation of pressure drop is clearly essential to reproduce the observed pressure drop across the MCHE of about 1.8 MPa (Figure 8).

Figure 9 shows the results of the CryoFAST simulation of RasGas LNG Train 4 for the normal operating condition. The calculated pressure drop along the length of the MCHE is highlighted in Figure 9a by presenting the results obtained with and without consideration of 
centrifugal and twisting effects through eqs (17) to (21). For an estimated total tube length of $100 \mathrm{~m}$, the predicted pressure drop across the MCHE is less than $0.2 \mathrm{MPa}$ if these effects are not considered, while their inclusion leads to a calculated pressure drop of $2.0 \mathrm{MPa}$, which is consistent with the operating data shown in Figure 8 prior to August 2014. This result emphasises the importance of incorporating centrifugal and twisting forces into pressure drop calculations for CWHEs used in LNG production. At around $76 \mathrm{~m}$, the natural gas passes through its bubble point (Figure 9(c)), converting to a compressed liquid from a two phase vapour-liquid mixture. The cusp in the temperature profile of the fluid shown in Figure 9(d) at this point reflects the transition from latent to sensible cooling of the mixture.
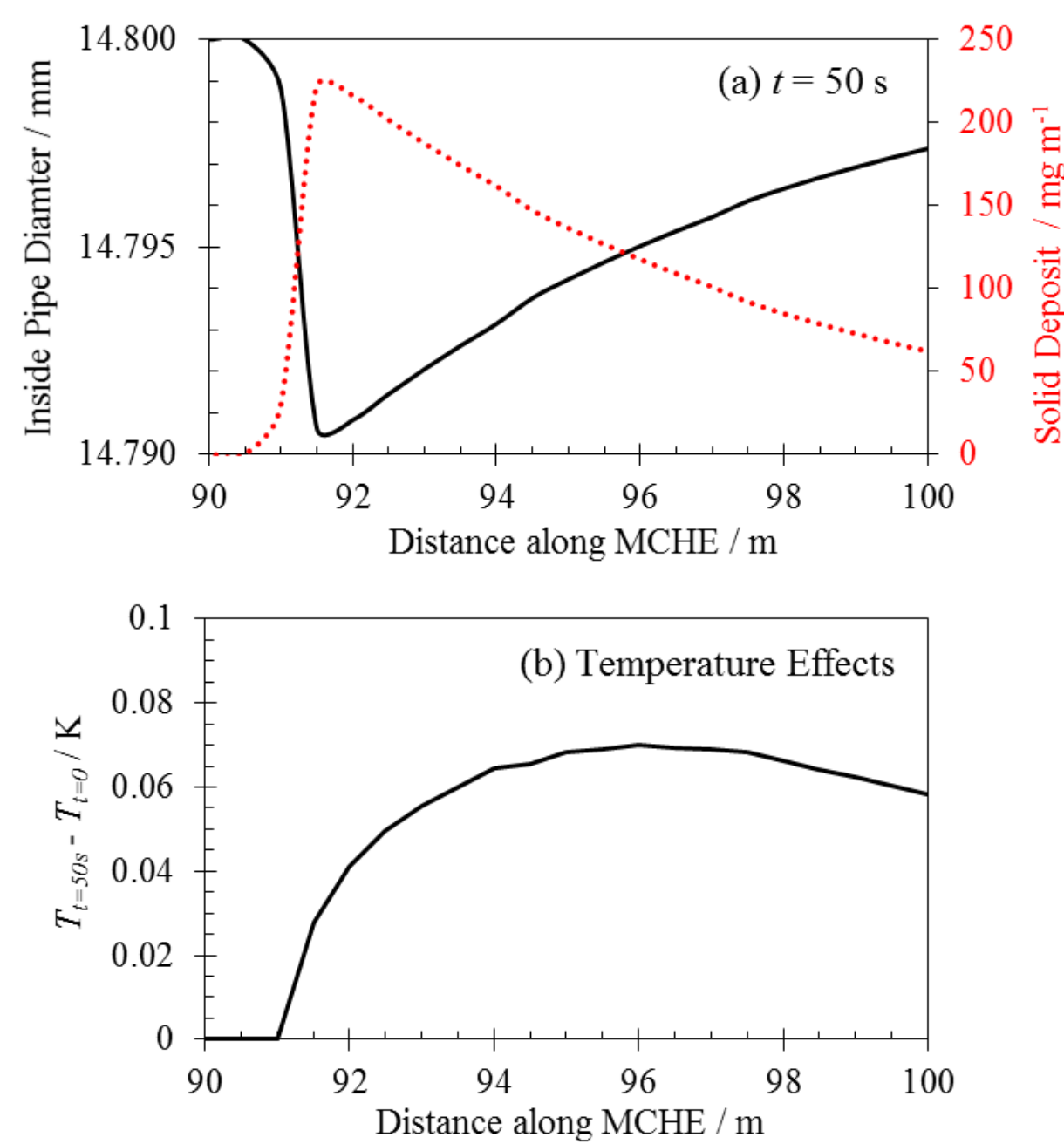

Figure 10: The key result of the Upset Operating Condition simulation under transient conditions as a function of position in the main cryogenic heat exchanger (MCHE). (a) Total expected benzene deposition (red dotted curve) following the re-start due to the carryover for $50 \mathrm{~s}$ of $0.2 \mathrm{~mol}^{2} \mathrm{C}_{5+}$ and the effect it had on the effective internal diameter of each tube (solid black curve). (b) Change in LNG temperature within the MCHE after $50 \mathrm{~s}$ : for a 
constant duty in the refrigeration cycle, the benzene deposit would have caused an increase in the effluent LNG temperature of approximately $0.06 \mathrm{~K}$.

Figure 10a shows the key result of the Upset Operating Condition simulation: solid benzene deposits at a location nearly $90 \%$ of the way through the CWHE, causing the internal diameter of the tubing at that location to decrease by about $0.2 \%$. This location is consistent with the observations of Ismail and Al Thani [30], who reported the blockage to be in the upper cold bundle of the CWHE. The first moment of deposition occurred 23 seconds after the upset feed reached the MCHE inlet. The simulation results indicate that the total amount of benzene deposited per tube was only $1.2 \mathrm{~g}$ as a result of 50 seconds of heavy carry-over or $1.9 \mathrm{~kg}$ for the entire MCHE. This is approximately $75 \%$ of the total amount of benzene that would have passed through the exchanger during this short period of time (with the balance remaining in the LNG solution). Consequently, the deposit's impact on the operating data is negligible and, consistent with the results shown by Ismail and $\mathrm{Al}$ Thani [30], detection of its presence at this time could not be expected from measurements of pressure drop, especially considering the signal to noise of plant data. The simulation results show that maximum relative changes to total pressure drop and mass flow rate would have been only $+0.002 \%$ and $-0.03 \%$, respectively, which are essentially unmeasurable in an operating plant environment. The benzene deposition predicted by the simulation would have also increased the heat transfer resistance between the refrigerant and the wall. The predicted consequence of such a deposition is either the effluent temperature would have increased by about $0.06 \mathrm{~K}$ (if the refrigerant duty were constant) as shown in Figure 10b, or the refrigerant duty would have increased by $0.03 \%$ (if the effluent temperature were controlled, which is more likely). Given their small magnitude, such changes would also have been undetectable by plant operators. However, if heavy carry-over had continued for 10 minutes then while the fractional changes in pressure drop and mass flow rate would still have only been $+0.03 \%$ and $-0.03 \%$, respectively, the change in effluent temperature or the refrigerant duty would have been about $+0.9 \mathrm{~K}$ or $0.4 \%$, respectively. This suggests that changes in effluent temperature or refrigerant duty may provide a better initial indication of deposition in heat exchangers than changes in flow rate or pressure drop.

This initial small change in pressure drop explains why the plant operators had to wait over two months before they could unambiguously diagnose that a problem had occurred. In many respects, however, that latency proved to be the reason why an unplanned shut down became necessary to rectify the problem. Ismail and Al Thani [30] hypothesise that the initial 
deposition of benzene provided a site for water molecules in the gas phase to nucleate, adsorb and accumulate, even though the moisture content of the feed gas always remained below the 1 ppm specification (see Figure 8(a)). Ultimately about 100 kilograms of liquid water was removed from the MCHE upon thawing in December 2014, which suggests that about $60 \%$ of the water entering the heat exchanger over that four month period (at an average vapour concentration around $0.6 \mathrm{ppm}$ ) deposited on or around the nucleation site provided initially by the frozen benzene.

In an attempt to assess the hypothesis of Ismail and Al Thani [30], we calculated the equilibrium temperatures for both hydrate and ice phases for the LNG compositions shown in Table 1 in the presence of $0.5 \mathrm{ppm}$ of $\mathrm{H}_{2} \mathrm{O}$ at a total pressure of about $3 \mathrm{MPa}$. To our knowledge no experimental data for ice or hydrate formation are available at such low temperatures $(\approx 140 \mathrm{~K})$ and water concentrations, requiring the significant extrapolation of any model used to make such predictions. An ice equilibrium temperature of $207 \mathrm{~K}$ was predicted using eqs (1) - (3) as implemented in ThermoFAST. An alternative estimate can be made by estimating the fugacity of pure ice from its vapour pressure, which can be accurately calculated using the reference equation of Wagner et al. [60]. For a gas pressure of $3.2 \mathrm{MPa}$ and a water mole fraction of $0.5 \times 10^{-6}$, the vapour fugacity of $\mathrm{H}_{2} \mathrm{O}$ could be approximated using the ideal-gas law as $1.6 \mathrm{~Pa}$, giving an equilibrium temperature of $216 \mathrm{~K}$. The hydrate equilibrium condition can also be estimated in a variety of ways. The cubic plus association

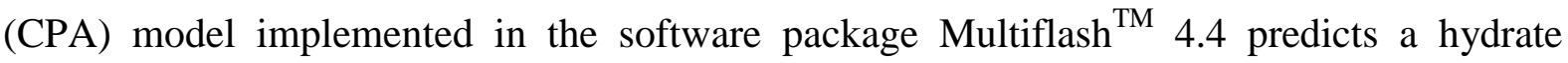
equilibrium temperature of $218 \mathrm{~K}$. Makogon and Sloan [61] reported methane hydrate equilibrium data down to $148 \mathrm{~K}$ and we estimate that for the measured equilibrium condition at $(208 \mathrm{~K}, 0.22 \mathrm{MPa})$ the vapour-phase water mole fraction would be $2 \mathrm{ppm}$ based on the vapour pressure of ice at this temperature. Thus the hydrate equilibrium pressure at this temperature if the vapour-phase water mole fraction were only $0.5 \mathrm{ppm}$ would be approximately $1 \mathrm{MPa}$, which is still well below the typical process pressure in an LNG Train.

These estimates of the water crystallisation equilibrium temperature at $0.5 \mathrm{ppm}$ all suggest that the RasGas LNG Train 4 (and others like it) routinely operate successfully without any detectable water crystallisation at subcoolings in excess of $80 \mathrm{~K}$ ! Clearly, additional research is needed to (i) confirm the equilibrium temperature estimates at such low water concentrations, (ii) establish if kinetic limitations prevent the formation despite the large driving force, and/or (iii) whether deposition is simply (and fortuitously) avoided as a result of the hydrodynamic forces acting on the micro-crystals of water. 
Nevertheless, the empirical evidence from Ismail and Al Thani [30] suggests that the localised benzene deposit in the MCHE was sufficient to trigger the deposition of a solid aqueous phase. Unfortunately, no simulation tool, including CryoFAST, can yet represent such complex phenomena. However, as shown below, the application of CryoFAST to analyse the initial deposition event can provide insight into the both the window of opportunity operators have to rectify the problem's root cause, and the likely efficacy of various possible remedial actions that could be applied during this window.

\subsection{Remedial Actions and Window of Opportunity}

Under normal operation, benzene should not solidify in the MCHE because, as shown in Figure 11, its concentration in the feed is less than its solubility in the MCHE effluent. Therefore, once normal operating conditions resumed following the RasGas LNG Train 4 start-up, the $1.2 \mathrm{~g}$ of deposited benzene per tube should have simply dissolved back into solution; this may be why the operators were not concerned with the brief increase in $\mathrm{C}_{5+}$ concentration above the specified limit. Simulations with CryoFAST indicate that complete dissolution of the benzene deposit should have taken less than 3 hours if the operators took no additional remedial action. Clearly this did not happen, which suggests that within these 3 hours enough water nucleated and/or adsorbed to the benzene deposit to enable the crystallisation of water to become self-sustaining, leading to the accumulation of about $100 \mathrm{~kg}$ in the MCHE over 4 months. Assuming that the rate of water crystallisation was constant through the 4 month period, approximately $0.09 \mathrm{~g}$ of ice per tube or $140 \mathrm{~g}$ over the entire MCHE would have deposited in the 3 hours following the benzene freeze out. This implies that the window of opportunity to remediate the problem by dealing with the benzene deposit was, at most, 3 hours. This is still well before the operating data provided any indication of a problem; however, a sufficiently rapid simulation to assess the likely risk associated with the scrub column's loss of reflux during start-up could potentially have indicated that some immediate action might be advisable. Any remedial action taken after the 3 hours would need to have focussed on removing the crystallised water. By November 2014, the efficacy of the remedial action taken by the operators proved to be insufficient. 


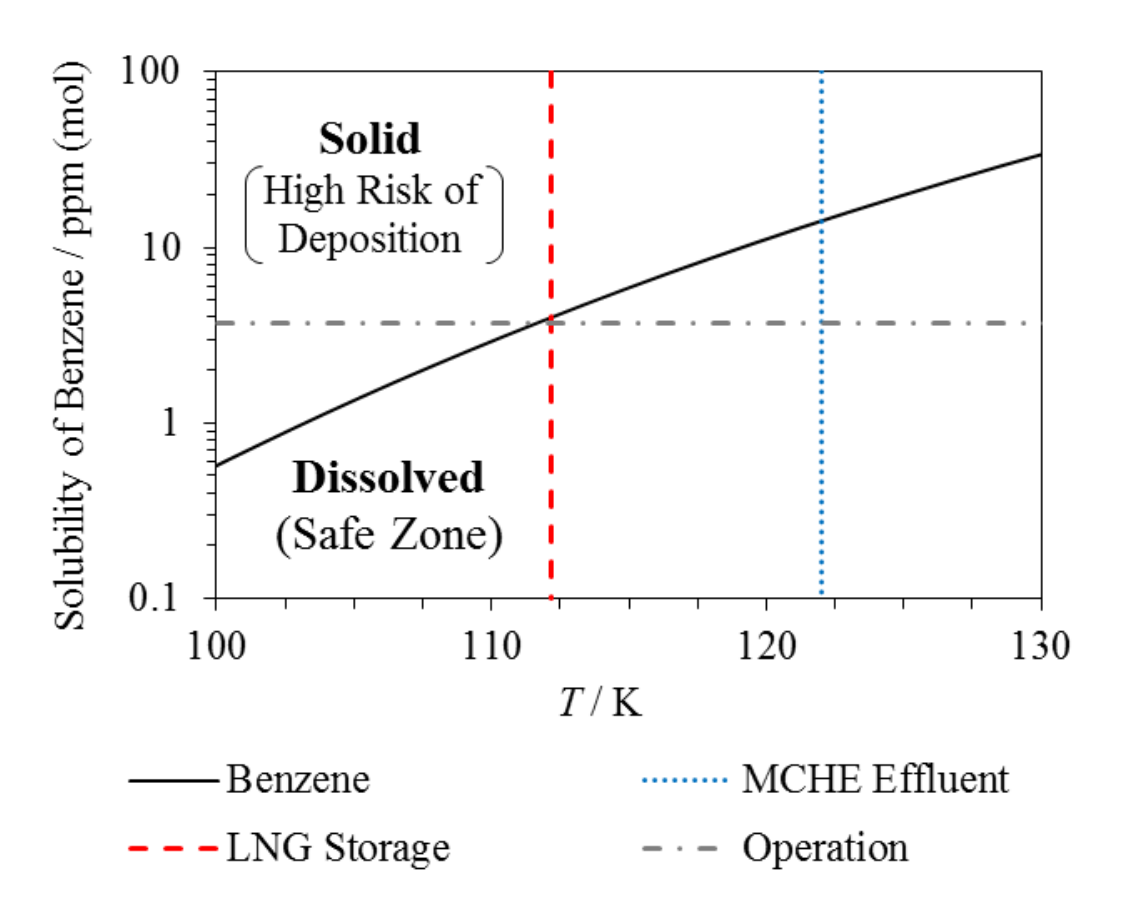

Figure 11: Solubility of benzene at $5 \mathrm{MPa}$ in the RasGas LNG mixture under normal operating conditions showing the lowest temperature in the MCHE cold bundle (i.e. MCHE effluent temperature), the LNG storage temperature downstream of the final letdown (flash) valve ($161{ }^{\circ} \mathrm{C}$ or $\left.112.15 \mathrm{~K}\right)$, and the operating composition of benzene under normal conditions.

Three possible remedial actions to remove the benzene deposit were considered here using CryoFAST simulations: (i) increase the temperature of the MCHE cold bundle to accelerate the dissolution of the solid; (ii) temporarily drive the upstream distillation (scrub) column's condenser harder to create a leaner fluid composition entering the MCHE; or (iii) a combination of (i) and (ii). For case (i) the MCHE's effluent temperature was increased by 2 $\mathrm{K}$ by increasing the temperature of the refrigerant in the latter sections of the MCHE. Such a local temperature increase was applied to the MCHE cold bundle in November 2014 by the RasGas LNG operators, and was observed to partially shift some of the (large) accumulated deposit. If this action had been applied immediately following the initial deposition, the increase in benzene solubility from $4.0 \mathrm{ppm}$ to $5.3 \mathrm{ppm}$ in the LNG would have reduced the time taken to re-dissolve the deposit from 3 hours to 31 minutes.

For case (ii), a reduction in the concentration of $\mathrm{C}_{5+}$ entering the MCHE from $0.1 \mathrm{~mol} \%$ to $0.05 \mathrm{~mol} \%$ was considered, without any change to the temperature and pressure profiles within the heat exchanger. The resulting reduction in the feed's benzene concentration from $3.7 \mathrm{ppm}$ to $1.9 \mathrm{ppm}$ reduces the time required to remove the initial deposit to 23 minutes. Combining both remedial actions, as was done for case (iii), meant that the LNG in the vicinity of the initial deposit had a benzene concentration of just $1.9 \mathrm{ppm}$ with a solubility 
limit of $5.3 \mathrm{ppm}$. Under these circumstances, the initial $1.2 \mathrm{~g}$ of deposited benzene would be removed in just 14 minutes (a $92 \%$ reduction in time), substantially reducing the risk of any water crystallisation becoming self-sustaining.

\section{Conclusion}

In this work, the RasGas LNG Train 4 operational incident described by Ismail and $\mathrm{Al}$ Thani [30] was simulated for the first time using two new tools, CryoFAST and ThermoFAST. The latter is a thermodynamic engine based on the Peng Robinson equation of state tuned to the available data for heavy hydrocarbon solubility in methane or ethane. We demonstrate that this thermodynamic model is able to represent solid liquid equilibria as accurately as the KLSSP tool used widely by industry over a wider range of compositions and pressures. The CryoFAST model combines such thermodynamic calculations with the approximate but efficient solution of dynamic material and energy balance equations, and empirical pressure drop correlations for multi-phase flow which include important contributions for centrifugal and twisting forces in CWHEs.

The operational data from RasGas LNG Train 4 indicate that over 2 months were required before the presence of a solid deposit could be unambiguously determined. In contrast the simulation predicted correctly the location of the initial benzene deposit that occurred upon restart within the Main Cryogenic Heat Exchanger. When combined with the operational record, the simulation reveals that the operators had only a short window of opportunity to remedy the situation before a blockage requiring shutdown of the plant became inevitable. Possible methods of remediating the initial benzene deposit within this window of opportunity were explored: heating the MCHE cold bundle by $2 \mathrm{~K}$ and using scrub column condenser to reduce the feed's benzene concentration may have removed the deposit in 14 minutes.

\section{Acknowledgements}

This research was supported by the Australian Research Council through the Industrial Transformation Training Centre for LNG Futures, IC150100019. The authors thank Paul Pickering for guidance with the modelling of centrifugal and twisting effects on flow. We are also grateful to Stanley Huang and Dan McCartney and GPA Midstream for help with the development of ThermoFAST and literature data comparisons. 


\section{References}

[1] BP. BP Statistical Review of World Energy June 2016. 2016,

https://www.bp.com/content/dam/bp/pdf/energy-economics/statistical-review-2016/bp-statistical-review-ofworld-energy-2016-full-report.pdf [accessed 15.05.2017].

[2] International Gas Union. Small Scale LNG. 2015, http://www.igu.org/sites/default/files/node-pagefield file/SmallScaleLNG.pdf [accessed 15.05.2017].

[3] International Gas Union. World LNG Report. 2016, www.igu.org/download/file/fid/2123 [accessed 15.05.2017].

[4] Visser A. World Fleet of LNG Carriers > 75.000 m3. 2012, http://www.aukevisser.nl/supertankers/gas-

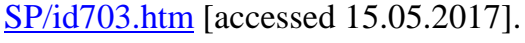

[5] Ministry of Economy Trade and Industry. Trend of the price of spot-LNG. 2017, http://www.meti.go.jp/english/statistics/sho/slng/result/pdf/201704-e.pdf [accessed 15.05.2017].

[6] Baccanelli M, Langé S, Rocco MV, Pellegrini LA, Colombo E. Low temperature techniques for natural gas purification and LNG production: An energy and exergy analysis. Appl Energy. 2016;180:546-59.

[7] Khan MS, Lee S, Rangaiah GP, Lee M. Knowledge based decision making method for the selection of mixed refrigerant systems for energy efficient LNG processes. Appl Energy. 2013;111:1018-31.

[8] Mortazavi A, Somers C, Hwang Y, Radermacher R, Rodgers P, Al-Hashimi S. Performance enhancement of propane pre-cooled mixed refrigerant LNG plant. Appl Energy. 2012;93:125-31.

[9] Madouri A. Improvement of high heating value of commercialised liquefied natural gas of GL1Z plant by optimising the LPG extraction. LNG-14, Doha, Qatar 2004.

[10] Dauber F, Span R. Achieving higher accuracies for process simulations by implementing the new reference equation for natural gases. Comput Chem Eng. 2012;37:15-21.

[11] Dauber F, Span R. Modelling liquefied-natural-gas processes using highly accurate property models. Appl Energy. 2012;97:822-7.

[12] Kunz O, Wagner W. The GERG-2008 Wide-Range Equation of State for Natural Gases and Other Mixtures: An Expansion of GERG-2004. J Chem Eng Data. 2012;57:3032-91.

[13] May EF, Guo JY, Oakley JH, Hughes TJ, Graham BF, Marsh KN, et al. Reference Quality Vapor-Liquid Equilibrium Data for the Binary Systems Methane + Ethane, + Propane, + Butane, and + 2-Methylpropane, at Temperatures from (203 to 273) K and Pressures to 9 MPa. J Chem Eng Data. 2015;60:3606-20.

[14] Rowland D, Hughes TJ, May EF. Extending the GERG-2008 equation of state: Improved departure function and interaction parameters for (methane + butane). J Chem Thermodyn. 2016;97:206-13.

[15] Norris BWE, Zerpa LE, Koh CA, Johns ML, May EF, Aman ZM. Rapid assessments of hydrate blockage risk in oil-continuous flowlines. J Nat Gas Sci Eng. 2016;30:284-94.

[16] Aman ZM, Zerpa LE, Koh CA, Sum AK. Development of a Tool to Assess Hydrate-Plug-Formation Risk in Oil-Dominant Pipelines. Soc Pet Eng J. 2015;20:884-92.

[17] Kohn JP, Luks KD. Solubility of Hydrocarbons in Cryogenic LNG and NGL Mixtures. GPA Research Report 22; 1976.

[18] Kohn JP, Luks KD, Liu PH. Three-phase solid-liquid-vapor equilibriums of binary-n-alkane systems (ethane-n-octane, ethane-n-decane, ethane-n-dodecane). J Chem Eng Data. 1976;21:360-2.

[19] Kohn JP, Luks KD, Liu PH, Tiffin DL. Three-phase solid-liquid-vapor equilibriums of the binary hydrocarbon systems methane-n-octane and methane-cyclohexane. J Chem Eng Data. 1977;22:419-21. 
[20] Liu PH, Luks KD, Kohn JP. Three-phase solid-liquid-vapor equilibria of the systems ethane-benzene, ethane-cyclohexane, and ethane-trans-decalin. J Chem Eng Data. 1977;22:220-1.

[21] Tiffin DL, Kohn JP, Luks KD. Three-phase solid-liquid-vapor equilibriums of binary ethylene-n-alkane systems (ethylene-n-octane, ethylene-n-decane, ethylene-n-dodecane). J Chem Eng Data. 1978;23:207-9.

[22] Tiffin DL, Kohn JP, Luks KD. Solid hydrocarbon solubility in liquid methane-ethane mixtures along threephase solid-liquid-vapor loci. J Chem Eng Data. 1979;24:306-10.

[23] Tiffin DL, Kohn JP, Luks KD. Three-phase solid-liquid-vapor equilibriums of the systems ethylenecyclohexane, ethylene-trans-decalin, ethylene-benzene, and ethylene-2-methylnaphthalene. J Chem Eng Data. 1979;24:96-8.

[24] Tiffin DL, Kohn JP, Luks KD. Three phase solid-liquid-vapor equilibriums of the binary hydrocarbon systems ethane-2-methylnaphthalene, ethane-naphthalene, propane-n-decane, and propane-n-dodecane. J Chem Eng Data. 1979;24:98-100.

[25] Chen W-L, Callahan SF, Luks KD, Kohn JP. Effect of the presence of nitrogen on solid solubility of normal paraffins in liquid methane. J Chem Eng Data. 1981;26:166-8.

[26] Chen W-L, Luks KD, Kohn JP. Three-phase solid-liquid-vapor equilibriums of the binary hydrocarbon systems propane-benzene, propane-cyclohexane, butane-benzene, butane-cyclohexane, butane-n-decane, and butane-n-dodecane. J Chem Eng Data. 1981;26:310-2.

[27] Hottovy JD, Kohn JP, Luks KD. Partial miscibility behavior of the methane-ethane-n-octane system. J Chem Eng Data. 1981;26:135-7.

[28] Luks KD, Hottovy JD, Kohn JP. Three-phase solid-liquid-vapor equilibriums in the binary hydrocarbon systems methane-n-hexane and methane-benzene. J Chem Eng Data. 1981;26:402-3.

[29] Hottovy JD, Kohn JP, Luks KD. Partial miscibility behavior of the ternary systems methane-propane-noctane, methane-n-butane-n-octane, and methane-carbon dioxide-n-octane. J Chem Eng Data. 1982;27:298-302.

[30] Ismail SM, Al-Thani K. Unique Phenomenon of Moisture \& Other Contaminants' Coalescence under Cryogenic Conditions. LNG-18, Perth, Australia 2016.

[31] Peng D-Y, Robinson DB. A New Two-Constant Equation of State. Ind Eng Chem. 1976;15:59-64.

[32] Hildebrand JH, Scott RL. The Solubility of Nonelectrolytes. New York: Reinhold Pub. Corp.; 1950.

[33] Prausnitz JM, Lichtenthaler RN, de Azevedo EG. Molecular Thermodynamics of Fluid-Phase Equilibria: Pearson Education; 1998.

[34] Brown TS, Niesen VG, Erickson DD, Inc. C. The Effects of Light Ends and High Pressure on Paraffin Formation. SPE. 1994;28505.

[35] Lira-Galeana C, Firoozabadi A, Prausnitz JM. Thermodynamics of wax precipitation in petroleum mixtures. AIChE Journal. 1996;42:239-48.

[36] Morgan DL, Kobayashi R. Triple point corresponding states in long-chain n-alkanes. Fluid Phase Equilibria. 1991;63:317-27.

[37] Won KW. Thermodynamics for solid solution-liquid-vapor equilibria: wax phase formation from heavy hydrocarbon mixtures. Fluid Phase Equilibria. 1986;30:265-79.

[38] Kuebler GP, McKinley C. Solubility of solid benzene, toluene, n-hexane and n-heptane in liquid methane. Adv Cryog Eng. 1974;9:320.

[39] Dickinson E, Knobler CM, Scott RL. Solid/Liquid Phase Equilibria in the Mixtures Methane + n-Hexane and Methane + n-Pentane. J Chem Soc. 1973;69:2179-87.

[40] Luks KD, Hottovy JD, Kohn JP. Three-Phase Solid-Liquid-Vapor Equilibria in the Binary Hydrocarbon Systems Methane- $n$-Hexane and Methane-Benzene. J Chem Eng Data. 1981;26:402-3. 
[41] Shim J, Kohn JP. Multiphase and Volumetric Equilibria of Methane- $n$-Hexane Binary System at Temperatures Between $-110^{\circ}$ and $150^{\circ}$ C. J Chem Eng Data. 1962;7:3-8.

[42] Nicklin DJ, Wilkes JO, Davidson JF. Two-Phase Flow in Vertical Tubes. Trans Inst Chem Eng. 1962;40:61-8.

[43] Bendiksen KH. An experimental investigation of the motion of long bubbles in inclined tubes. Int $\mathrm{J}$ Multiphase Flow. 1984;10:467-83.

[44] Sinnott RK, Coulson JM, Richardson JF. Chemical Engineering Design: Chemical Engineering. 4th ed. Oxford: Elsevier Science; 2005.

[45] Haj-Shafiei S, Serafini D, Mehrotra AK. A steady-state heat-transfer model for solids deposition from waxy mixtures in a pipeline. Fuel. 2014;137:346-59.

[46] DIPPR® Thermophysical Properties Laboratory. DIPPR® 801. AIChE. 2017. Accessed from: https://dippr.aiche.org/.

[47] Beggs HD, Brill JP. A study of two-phase flow in incline pipes. J Petroleum Tch. 1973;25:607-17.

[48] Finn AJJ, G. L.; Tomlinson, T. R. . Developments in Natural Gas Liquefaction. Hydrocarbon Process, Int Ed. 1999;78:47-59.

[49] Pickering PF. Bubbles Going Round the Twist. Gas-Liquid Flows in Vertical Small Amplitude Helical Tubes (SMAHT). Annual Multiphase Flow Club. Imperial College, London 2007.

[50] Jayanti S, Hewitt GF. On the paradox concerning friction factor ratio in laminar flow in coils. Proc R Soc Lond A. 1991:291-9.

[51] Jayanti S, Hewitt GF. Prediction of onset of nucleate boiling, net vapour generation and critical heat flux in coiled tubes. Warme- und Staffebertragung. 1991:301-5.

[52] Jayanti S, Hewitt GF. A numerical study of bifurcation in laminar flow in curved ducts. Int J Numer Methods Fluids. 1992:253-66.

[53] Jayanti S, Hewitt GF. Prediction of film inversion in two-phase flow in coils. J Fluid Mech. 1992:497-511.

[54] Colebrook CF, White CM. Experiments with Fluid Friction in Roughened Pipes. Proc R Soc Lond A. 1937;161:367-78.

[55] Al-Muhannadi MHM, Okuyama MT, Durr MC. Successful completion and initial operation of the Ras Laffan onshore facilities (LNG) project. Ras Laffan Liquefied Natural Gas Company; 2000.

[56] Truong T, Kinard GE, Liu YN. Coil Wound Heat Exchanger. In: Air Products And Chemicals I, editor. European Patent 2003.

[57] Mølnvik MJ. Compact LNG Heat Exchangers. 2003, www.forskningsradet.no/csstorage/vedlegg/156662.ppt [accessed 13.02.2016].

[58] RasGas Company Limited. Trains 3, 4 and 5. 2013, http://rasgas.com/Operations/Trains34And5.html [accessed 15.06.2016].

[59] Neeraas BO. Tube Side Heat Transfer of Condensing Hydrocarbons in Coil-Wound LNG Heat Exchanger. International Heat Transfer Conference. Brighton, UK 2014. p. 127.

[60] Wagner W, Riethmann T, Feistel R, Harvey AH. New Equations for the Sublimation Pressure and Melting Pressure of H2O Ice Ih. Journal of Physical and Chemical Reference Data. 2011;40:043103.

[61] Makogon TY, Sloan ED, Jr. Phase Equilibrium for Methane Hydrate from 190 to 262 K. Journal of Chemical \& Engineering Data. 1994;39:351-3. 


\section{Nomenclature}

\section{Glossary}

$D \quad$ Diameter of the pipe or tube [m]

De Dean Number

$\mathrm{Fr}_{N} \quad$ Froude number

Ge Germano Number

$L \quad$ Total liquid mole fraction

$P \quad$ Pressure $[\mathrm{Pa}]$

$Q \quad$ Heat transfer to the environment [J]

$R \quad$ Tube's radius [m]

$T \quad$ Temperature $[\mathrm{K}]$

$U \quad$ Overall heat transfer coefficient $\left[\mathrm{W} \mathrm{m}^{-2} \mathrm{~K}^{-1}\right]$

$V \quad$ Overall vapour mole fraction

$V \quad$ Volume of the node $\left[\mathrm{m}^{3}\right]$

$g \quad$ Acceleration due to the gravity $\left[\mathrm{m} \mathrm{s}^{-2}\right]$

$n \quad$ Node number

$t \quad$ Time-step

$u \quad$ Overall fluid velocity $\left[\mathrm{m} \mathrm{s}^{-1}\right]$

$\beta \quad$ Vapour mole fraction for VLE only

$\rho \quad$ Density $\left[\mathrm{kg} \mathrm{m}^{-3}\right]$

$\chi \quad$ Solid deposition mole fraction (solids deposited / total solids formed)

$\delta \quad$ Radial thickness of the deposit [m]

$(U A)_{d e p} \quad$ Local heat transfer $\left[\mathrm{W} \mathrm{K}^{-1}\right]$

$\Lambda_{c} \quad$ Coil pitch [m]

$C_{D} \quad$ Distribution factor

$L_{n} \quad$ Node spacing length [m]

$N_{\text {nodes }} \quad$ Total number of nodes

$N_{t-\text { steps }} \quad$ Total number of time-steps

$P_{m} \quad$ Reference pressure at $T_{m, i}[\mathrm{~Pa}]$

$\dot{Q} \quad$ Rate of heat transfer to the environment $\left[\mathrm{J} \mathrm{s}^{-1}\right]$

$R_{c} \quad$ Radius of the coil bundle [m]

$S_{F} \quad$ Total solid phase mole fraction

$T_{m, i} \quad$ Melting temperature of pure component $i[\mathrm{~K}]$

$U_{N D} \quad$ Overall heat transfer coefficient in the absence of deposition [W m $\left.{ }^{-2} \mathrm{~K}^{-1}\right]$

$f_{i}^{s} \quad$ Fugacity of pure component $i$ in the solid phase [Pa]

$k_{\text {dep }} \quad$ Thermal conductivity of the deposit layer $\left[\mathrm{W} \mathrm{m}^{-1} \mathrm{~K}^{-1}\right]$

$\dot{m} \quad$ Mass flow rate $\left[\mathrm{kg} \mathrm{s}^{-1}\right]$

$m_{\text {dep }} \quad$ Total mass deposition $[\mathrm{kg}]$

$s_{i} \quad$ Solid phase mole fraction of component $i$

$u_{D} \quad$ Drift velocity in a stagnant fluid $\left[\mathrm{m} \mathrm{s}^{-1}\right]$

$u_{S L} \quad$ Superficial velocity of the liquid phase $\left[\mathrm{m} \mathrm{s}^{-1}\right]$

$u_{S V} \quad$ Superficial velocity of the vapour phase $\left[\mathrm{m} \mathrm{s}^{-1}\right]$

$u_{V} \quad$ Vapour phase velocity $\left[\mathrm{m} \mathrm{s}^{-1}\right]$

$w_{i} \quad$ Mass fraction of component $i$

$x_{i} \quad$ Liquid phase mole fraction of component $i$

$y_{i} \quad$ Vapour phase mole fraction of component $i$

$z_{H} \quad$ Vertical height [m]

$z_{i} \quad$ Overall composition of component $i$ 
$\varphi_{i}^{L} \quad$ Partial fugacity coefficient of component $i$ in the liquid mixture

$\varphi_{\text {pure }, i}^{L} \quad$ Fugacity coefficient of pure component $i$ in the liquid phase

$\phi_{V} \quad$ Vapour phase volume fraction

$\Delta h_{f u s, i} \quad$ Specific heat of fusion of each solid component deposited, $i\left[\mathrm{~J} \mathrm{~kg}^{-1}\right]$

$\Delta H_{f, i} \quad$ molar heat of fusion at $T_{m, i}[\mathrm{~J}]$

$\Delta T_{L M T D} \quad$ Log mean temperature difference $[\mathrm{K}]$

$\Delta c_{p, i}^{L \rightarrow S} \quad$ molar specific heat of the component as a pure liquid minus that of the pure solid, i [ $\mathrm{J} \mathrm{K}^{-1}$ ]

$\Delta v_{i}^{L \rightarrow S} \quad$ change in component's, $i$, volume between the liquid and solid phase $\left[\mathrm{m}^{3} \mathrm{~mol}^{-1}\right.$ ]

$\Delta P \quad$ Pressure Drop $[\mathrm{Pa}]$

$\Delta t \quad$ Length of time-step [s]

\section{Abbreviation, Acronyms}

BTEX Benzene, Toluene, Ethylbenzene, Xylene

$\mathrm{C}_{1} \quad$ Methane

$\mathrm{C}_{2} \mathrm{H}_{6} \quad$ Ethane

$\mathrm{C}_{3+} \quad$ Propane or Heavier Hydrocarbons

$\mathrm{C}_{3} \mathrm{H}_{8} \quad$ Propane

$\mathrm{C}_{4} \quad$ Butane

$\mathrm{C}_{5+} \quad$ Pentane or Heavier Hydrocarbons

$\mathrm{C}_{6} \mathrm{H}_{14} \quad$ Hexane

$\mathrm{C}_{6} \mathrm{H}_{6} \quad$ Benzene

$\mathrm{C}_{7} \mathrm{H}_{16} \quad$ Heptane

$\mathrm{C}_{7} \mathrm{H}_{8} \quad$ Toluene

$\mathrm{CH}_{4} \quad$ Methane

$\mathrm{CO}_{2} \quad$ Carbon Dioxide

CPA Cubic Plus Association

CWHE Coil Wound Heat Exchanger

GPA Gas Processors Association

$\mathrm{H}_{2} \mathrm{O} \quad$ Water

$\mathrm{iC}_{4} \mathrm{H}_{10} \quad$ Isobutane

$\mathrm{iC}_{5} \mathrm{H}_{12} \quad$ Isopentane

KL Kohn and Luks

KLSSP Kohn and Luks Solid Solubility Program

LNG Liquefied Natural Gas

LPG Liquefied Petroleum Gas

MCHE Main Cryogenic Heat Exchanger

$\mathrm{nC}_{4} \mathrm{H}_{10} \quad$ Normal Butane

$\mathrm{nC}_{5} \mathrm{H}_{12} \quad$ Normal Pentane

PR Peng and Robinson

SLE Solid Liquid Equilibrium

SLVE Solid Liquid Vapour Equilibrium

SVE Solid Vapour Equilibrium

VLE Vapour Liquid Equilibrium 